\title{
Stationary Response of Flag-shaped Hysteretic System under Combined Harmonic and White Noise Excitations
}

\section{L.C. Chen ( $\square$ lincongchen@hqu.edu.cn )}

Huaqiao University https://orcid.org/0000-0002-4563-9313

Huiying HU

huaqiao unversity

Shushen Ye

Huaqiao University

\section{Original Research}

Keywords: flag-shaped hysteresis, stochastic averaging method, combined Gaussian white noise and harmonic excitation, stochastic jump and P-bifurcation

Posted Date: February 17th, 2021

DOI: https://doi.org/10.21203/rs.3.rs-201883/v1

License: (c) (i) This work is licensed under a Creative Commons Attribution 4.0 International License. Read Full License 


\title{
Stationary response of flag-shaped hysteretic system under combined harmonic and white noise excitations
}

\author{
Huiying $\mathrm{Hu}^{1,2}$, Lincong Chen ${ }^{*}, 1,2$, Shushen $\mathrm{Ye}^{1}$ \\ ${ }^{1}$ College of Civil Engineering, Huaqiao University, 361021, Xiamen, China \\ ${ }^{2}$ Key Laboratory for Intelligent Infrastructure and Monitoring of Fujian Province, Huaqiao University, Jimei \\ Avenue 668, Xiamen, Fujian, 361021, China
}

\begin{abstract}
The dynamical system containing flag-shaped hysteretic behavior is common in practice. In this paper, the stationary response of flag-shaped hysteretic system excited by harmonic excitation as well as Gaussian white noise is determined with the technique of stochastic averaging. The reliability of the presented approach is demonstrated by relevant digital simulation. The stochastic jump under a certain combination of parameters is found. The stochastic P-bifurcation phenomenon, i.e., the disappearance or appearance of bimodal shape of stationary response, occurs concerning to the variation of system's parameters. Besides, the response of the system exposed to only harmonic excitation or non-resonance case is also examined for comparison, respectively. The numerical results show that the stationary amplitude response displays typical "soft" system behavior, and the deterministic jump may occur under pure harmonic excitation. Moreover, the non-resonance response is always weaker than that of resonant case.
\end{abstract}

Keywords: flag-shaped hysteresis; stochastic averaging method; combined Gaussian white noise and harmonic excitation; stochastic jump and P-bifurcation

\section{Introduction}

The hysteresis is very common in science or engineering fields when structural systems encounter with severe loads. The term hysteresis is utilized to illustrate the constitutive relationship of restoring force, which is a history-dependent piecewise continuous function [1-2]. Different types of theoretical models have been introduced for characterizing the hysteretic system, such as Preisach model, bilinear model and Bouc-Wen model, etc. Recently, a special type of hysteretic systems has shown great promise for eliminating residual drift and received extensive study [3-4]. These systems processing with specific flag shape hysteresis curve are named flag-shaped hysteretic system. So far, the flag-shaped hysteretic system has been applied to model many structural systems, such as the self-centering system [5] in civil engineering, and shape memory alloys [6] in material engineering. In this research, we inquire into the stationary response of the randomly excited flag-shaped hysteretic system.

On the other hand, many approximate methods have been proposed for predicting

\footnotetext{
* Corresponding author. Tel: +86-0592-6162698; E-mail address: lincongchen@hqu.edu.cn
} 
the response of the hysteretic system exposed to random excitations. They are the methods of equivalent linearization [7-11], explicit time-domain method [12], probability density evolution method [13-14], Gaussian closure technique [15], exponential-polynomial closure (EPC) method [16-17], dissipation energy balancing method [18], stochastic averaging [19-26], deep neural networks [27], Monte Carlo simulation [28], etc. Among methods mentioned above, the stochastic averaging method has the remarkable advantages in simplifying the equation of motion while retaining the main nonlinear behavior of system [29]. However, such method has rarely been used to the flag-shaped hysteretic system. Up to now, only the equivalent linearization method was the common method in the researches [30-31]. Furthermore, these researches available were limited to pure random excitation. Note that structural systems exposed to combined deterministic and random loadings are abounding in real engineering. In this regard, the flag-shaped hysteretic systems exist rich phenomenon like bimodal or bifurcations and should deserve more attention.

In this paper, we investigate the stochastic response of an emerging flag-shaped hysteretic system exposed to Gaussian white noise as well as harmonic excitations. Specifically, by decomposing the restoring force, the flag-shaped hysteretic system is approximated as an equivalent non-hysteretic system. Then, such equivalent non-hysteretic system is further derived to the averaged equations with respect to the amplitude and phase, which involves stochastic averaging process. Accordingly, the corresponding reduced FPK equation is then established and solved to yield the stationary response. Finally, the impact of system's critical parameters, including the yield displacement, post-yielding stiffness coefficient, the frequency and amplitude of harmonic excitation, as well ae the energy dissipation coefficient, are examined. Besides, the proposed analytical solutions and the Monte Carlo simulations are in good agreement.

\section{Averaged Itô equation}

2.1 Theoretical model of flag-shaped hysteretic system

The motion equation of a harmonic and Gaussian white noise excited flag-shaped hysteretic system reads,

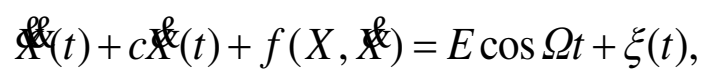

where $X$ is the displacement; $\xi(t)$ represents the Gaussian white noise excitation with intensity $2 D_{1} ; E$ and $\Omega$ denote the amplitude and frequency of harmonic excitation, 
respectively; $c$ represents the coefficient of damping. The term $f\left(X, X^{\&}\right)$ represents a flag-shaped restoring force (see Fig.1), which is made up of elastic force and hysteretic force. That is,

$$
f(X, k)=\alpha X+(1-\alpha) Z(X, k),
$$

in which $\alpha$ is the coefficient of post-yielding stiffness; hysteretic displacement $Z$ (see Fig.2) is given as,

$$
\begin{aligned}
& Z\left(X, X^{\&}\right)=\left\{\begin{array}{lr}
X-X_{y}+A & -A \leq X<-A+\beta X_{y} \\
-X_{y}+\beta X_{y} & -A+\beta X_{y} \leq X<-X_{y}+\beta X_{y} \\
X & -X_{y}+\beta X_{y} \leq X<X_{y} \\
X_{y} & X_{y} \leq X \leq A
\end{array}\right. \\
& Z\left(X, X^{\&}\right)=\left\{\begin{array}{lr}
-X_{y} & -A \leq X<-X_{y} \\
X & -X_{y} \leq X<X_{y}-\beta X_{y} \\
X_{y}-\beta X_{y} & X_{y}-\beta X_{y} \leq X<A-\beta X_{y} \\
X+X_{y}-A & A-\beta X_{y} \leq X \leq A
\end{array}, \quad \&<0 .\right.
\end{aligned}
$$

where $\beta$ is the energy dissipation coefficient; $A$ and $X_{y}$ are the amplitude and yield displacement of system, respectively.

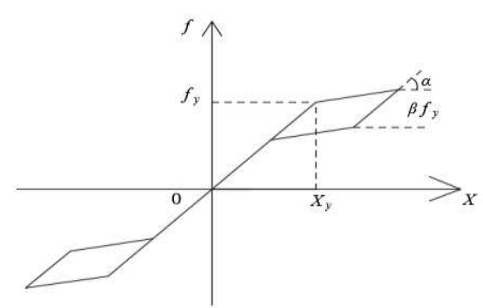

(a) The flag-shaped restoring force

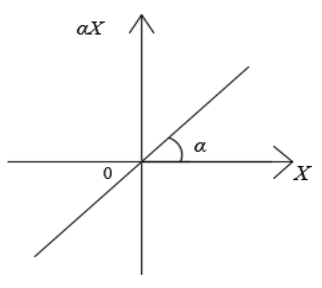

(b) The elastic force

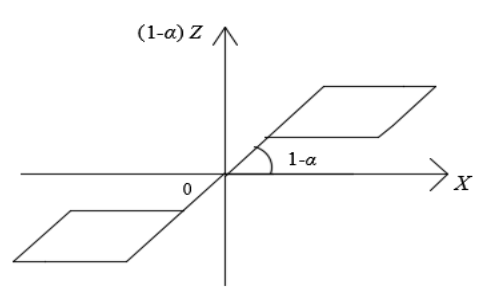

(c) The hysteretic force

Fig.1 The restoring force of the flag-shaped hysteretic system

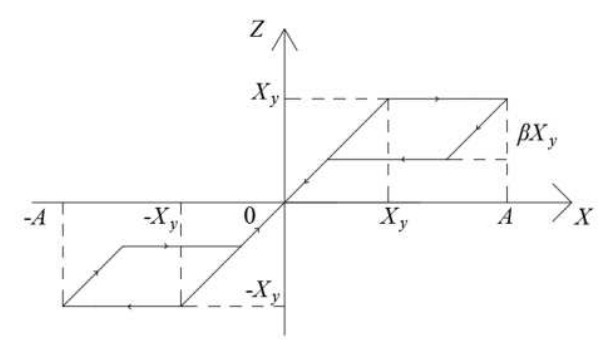

Fig.2 The hysteretic displacement of the flag-shaped hysteretic system 
2.2 Equivalent non-hysteretic system

Suppose that the response of the original system (1) is written as,

$$
\begin{aligned}
& X(t)=A(t) \cos \Theta^{\prime}(t), \\
& X^{\&}(t)=-A(t) \omega(A) \sin \Theta^{\prime}(t),
\end{aligned}
$$

in which

$$
\Theta^{\prime}(t) \approx \omega t+\varphi,
$$

$\omega$ denotes the averaged frequency of system (1).

Considering Fig.1, the flag-shaped hysteretic force can be decoupled into a combination of a quasi-linear elastic and a damping force, which can be realized by the technique of generalized harmonic balance, i.e.,

$$
f\left(X, X^{\&}\right)=K(A) X+C_{1}(A) X^{\&},
$$

in which $K(A)$ and $C_{1}(A)$ are called the equivalent coefficient of stiffness and damping, respectively. The expressions are given by,

$$
\begin{aligned}
C_{1}(A) & =-\frac{1}{\pi A \omega} \int_{0}^{2 \pi} f\left(X, X^{\&}\right) \sin \Theta^{\prime} \mathrm{d} \Theta^{\prime} \\
& =\left\{\begin{array}{ll}
0 & A \leq X_{y} \\
2(\alpha-1)\left(A-X_{y}\right) \beta X_{y} / \pi \omega A^{2} & A>X_{y}
\end{array},\right. \\
K(A) & =\frac{1}{\pi A} \int_{0}^{2 \pi} f(X, X) \cos \Theta^{\prime} \mathrm{d} \Theta^{\prime} \\
& =\left\{\begin{array}{ll}
1 & A \leq X_{y} \\
\left(\Omega_{1}+\Omega_{2}+\Omega_{3}+\Omega_{4}\right) / \pi & A>X_{y}
\end{array},\right.
\end{aligned}
$$

in which

$$
\begin{aligned}
& \Omega_{1}=\left[\sin \left(2 \theta_{2}\right)-\sin \left(2 \theta_{1}\right)\right] / 2+\left(\theta_{2}-\theta_{1}\right), \\
& \Omega_{2}=-\alpha\left[\sin \left(2 \theta_{2}\right)+2 \theta_{2}\right] / 2-2(1-\alpha) X_{y} \sin \theta_{2} / A, \\
& \Omega_{3}=\alpha\left\{\left[\sin \left(2 \theta_{1}\right)-\sin \left(2 \theta_{3}\right)\right] / 2+\theta_{1}-\theta_{3}\right\}+2(1-\alpha)(1-\beta)\left(\sin \theta_{1}-\sin \theta_{3}\right) X_{y} / A, \\
& \Omega_{4}=\sin \left(2 \theta_{3}\right) / 2+\theta_{3}+2(1-\alpha)\left(X_{y}-A\right) \sin \theta_{3} / A, \\
& \theta_{1}=\arccos \left[(1-\beta) X_{y} / A\right], \\
& \theta_{2}=\arccos \left(X_{y} / A\right), \\
& \theta_{3}=\arccos \left[\left(A-\beta X_{y}\right) / A\right] .
\end{aligned}
$$

Then the original system (1) characterized with flag-shaped restoring force is replaced by the following equivalent non-hysteretic system,

$$
(t)+\left[c+C_{1}(A)\right] X^{\&}(t)+K(A) X(t)=E \cos \Omega t+\xi(t),
$$

The total energy of the flag-shaped system is defined as,

$$
H=\frac{1}{2} X^{2}+\frac{1}{2} K(A) X^{2}=V(A),
$$

where 


$$
V(A)=\frac{1}{2} K(A) A^{2}
$$

2.3 Stochastic averaging

The response of the equivalent system (9) can be assumed as,

$$
\begin{aligned}
& X(t)=A(t) \cos \Theta(t), \\
& \&(t)=-A(t) \omega(A) \sin \Theta(t),
\end{aligned}
$$

in which

$$
\Theta(t) \approx \omega(A) t+\Phi(t) .
$$

Eq. (12) is taken to be the generalized van der Pol transformation, the equations of motion of $\left[X, X^{\&}\right]^{T}$ can be converted into,

$$
\begin{aligned}
& \frac{\mathrm{d} A}{\mathrm{~d} t}=F_{11}(A, \Theta)+F_{12}(A, \Theta, \Omega t)+G_{1}(A, \Theta) \xi(t), \\
& \frac{\mathrm{d} \Phi}{\mathrm{d} t}=F_{21}(A, \Theta)+F_{22}(A, \Theta, \Omega t)+G_{2}(A, \Theta) \xi(t),
\end{aligned}
$$

where

$$
\begin{aligned}
& F_{11}=-A\left[c+C_{1}(A)\right] \sin ^{2} \Theta, \\
& F_{12}=-\frac{\sin \Theta}{\omega} E \cos \Omega t, \\
& F_{21}=-\left[c+C_{1}(A)\right] \sin \Theta \cos \Theta, \\
& F_{22}=-\frac{\cos \Theta}{A \omega} E \cos \Omega t, \\
& G_{1}=-\frac{\sin \Theta}{\omega} \\
& G_{2}=-\frac{\cos \Theta}{\omega A}
\end{aligned}
$$

By adding Wong-Zakai correction terms, Eq. (14) can be converted into Itô stochastic differential equations as follows:

$$
\begin{aligned}
& \mathrm{d} A=\left(F_{11}(A, \Theta)+F_{12}(A, \Theta, \Omega t)+F_{13}(A, \Theta)\right) \mathrm{d} t+\sigma_{1}(A, \Theta) \mathrm{d} B(t), \\
& \mathrm{d} \Phi=\left(F_{21}(A, \Theta)+F_{22}(A, \Theta, \Omega t)+F_{23}(A, \Theta)\right) \mathrm{d} t+\sigma_{2}(A, \Theta) \mathrm{d} B(t),
\end{aligned}
$$

where

$$
\begin{gathered}
F_{13}=D_{1}\left(\frac{\sin ^{2} \Theta}{2 \omega^{4}} \frac{\mathrm{d} K(A)}{\mathrm{d} A}+\frac{\cos ^{2} \Theta}{A \omega^{2}}\right), \\
F_{23}=D_{1}\left(\frac{\sin \Theta \cos \Theta}{A^{2} \omega^{2}}+\frac{\sin \Theta \cos \Theta}{2 A \omega^{4}} \frac{\mathrm{d} K(A)}{\mathrm{d} A}-\frac{\sin \Theta \cos \Theta}{A^{2} \omega^{2}}\right), \\
\sigma_{1}^{2}=2 D_{1} \frac{\sin ^{2} \Theta}{\omega^{2}}, \\
\sigma_{2}^{2}=2 D_{1} \frac{\cos ^{2} \Theta}{A^{2} \omega^{2}} .
\end{gathered}
$$

and $B(t)$ is the unit Wiener process.

Since the existence of harmonic excitation, there are two cases of resonant and 
non-resonant should be identified. For the latter case, the harmonic excitation makes no difference in the first approximation. Assume that,

$$
\frac{\Omega}{\omega}=1+\varepsilon \sigma,
$$

where $\varepsilon \sigma$ denotes a small detuning parameter.

Resorting to the approximation relation in Eq. (13) and multiplying Eq. (18) with $t$, it leads to,

$$
\Omega t=\Theta-\Phi+\varepsilon \sigma \tau,
$$

where $\tau=\omega t$.

Introducing the new variable $\Gamma$, i.e.,

$$
\Gamma=\varepsilon \sigma \tau-\Phi .
$$

Then, Eq. (19) is transformed into,

$$
\Omega t=\Theta+\Gamma .
$$

Taking into account the Eq. (21) as a transformation from $\Phi$ to $\Gamma$, the Itô stochastic differential equations can be rewritten as,

$$
\begin{aligned}
& \mathrm{d} A=\left[F_{11}(A, \Theta)+F_{12}(A, \Theta, \Theta+\Gamma)+F_{13}(A, \Theta)\right] \mathrm{d} t+\sigma_{1}(A, \Theta) \mathrm{d} B(t), \\
& \mathrm{d} \Gamma=-\left[F_{21}(A, \Theta)+F_{22}(A, \Theta, \Theta+\Gamma)+F_{23}(A, \Theta)-\Omega+\omega\right] \mathrm{d} t-\sigma_{2}(A, \Theta) \mathrm{d} B(t) .
\end{aligned}
$$

Considering of the light damping and excitation of the original system, and averaging with respect to $\Theta$ from 0 to $2 \pi$, a two-dimensional diffusion Markov process for amplitude $A$ and phase $\Gamma$ is given below,

$$
\begin{aligned}
& \mathrm{d} A=\bar{m}_{1}(A, \Gamma) \mathrm{d} t+\bar{\sigma}_{1}(A, \Gamma) \mathrm{d} B(t), \\
& \mathrm{d} \Gamma=\bar{m}_{2}(A, \Gamma) \mathrm{d} t+\bar{\sigma}_{2}(A, \Gamma) \mathrm{d} B(t) .
\end{aligned}
$$

where,

$$
\begin{aligned}
& \bar{m}_{1}=-\frac{\left[c+C_{1}(A)\right] A}{2}+\frac{D_{1}}{4 \omega^{4}} \frac{\mathrm{d} K(A)}{\mathrm{d} A}+\frac{D_{1}}{2 A \omega^{2}}+\frac{\sin \Gamma}{2 \omega} E, \\
& \bar{m}_{2}=\frac{\cos \Gamma}{2 A \omega} E+\Omega-\omega, \\
& \bar{\sigma}_{1}^{2}=\frac{D_{1}}{\omega^{2}}, \bar{\sigma}_{2}^{2}=\frac{D_{1}}{A^{2} \omega^{2}} .
\end{aligned}
$$

The reduced FPK equation related to averaged Itô Eq. (23) can be written as,

$$
0=-\frac{\partial\left(\bar{m}_{1} p\right)}{\partial A}-\frac{\partial\left(\bar{m}_{2} p\right)}{\partial \Gamma}+\frac{1}{2} \frac{\partial^{2}\left(\bar{\sigma}_{1}^{2} p\right)}{\partial A^{2}}+\frac{1}{2} \frac{\partial^{2}\left(\bar{\sigma}_{2}^{2} p\right)}{\partial \Gamma^{2}},
$$

in which $p=p(A, \Gamma)$ is the stationary joint probability density. Given that $p(A, \Gamma)$ is the periodic function of $\Gamma$, it should satisfy the boundary condition as follows,

$$
p(A, \Gamma)=p(A, \Gamma+2 \pi),
$$

In addition, the boundary conditions for $A$ are, 


$$
\begin{aligned}
& p=\text { finite as } A=0, \\
& p=0, \partial p / \partial A=0 \text { as } A \rightarrow \infty,
\end{aligned}
$$

Moreover, Eq. (25) meets the following normalization condition,

$$
\int_{0}^{\infty} \int_{0}^{2 \pi} p(A, \Gamma) \mathrm{d} \Gamma \mathrm{d} A=1 .
$$

The reduced FPK Eq. (25) under the conditions (26)-(28) can be worked out numerically with the methods of successive over-relaxation and finite difference. After integration with respect to $\Gamma$, the stationary response of amplitude can be determined as follows,

$$
p(A)=\int_{0}^{2 \pi} p(A, \Gamma) \mathrm{d} \Gamma
$$

\section{Jump and bifurcation}

In this section, the special case of $D_{1}=0$ is considered firstly. That is to say, the system degenerates into deterministic excitation. The typical frequency response curves are shown in Fig.3. It is of interest to see that the curve tilt to the left side, and exhibit typical "soft" system behavior. Besides, the amplitude response of system is triple-valued within a certain frequency range of harmonic excitation. On the right boundary of the triple-valued region, the amplitude jumps from a larger value to a smaller one with the decrease of excitation frequency decreases. Inversely, on the left boundary, the amplitude jump from a smaller value to a larger one with the increase of excitation frequency. Such sudden change in amplitude is the deterministic jump phenomenon. Note that the phenomena of typical "soft" system behavior and deterministic jump obtained by the proposed technique are similar as those in Ref. [5] that used with averaging technique.

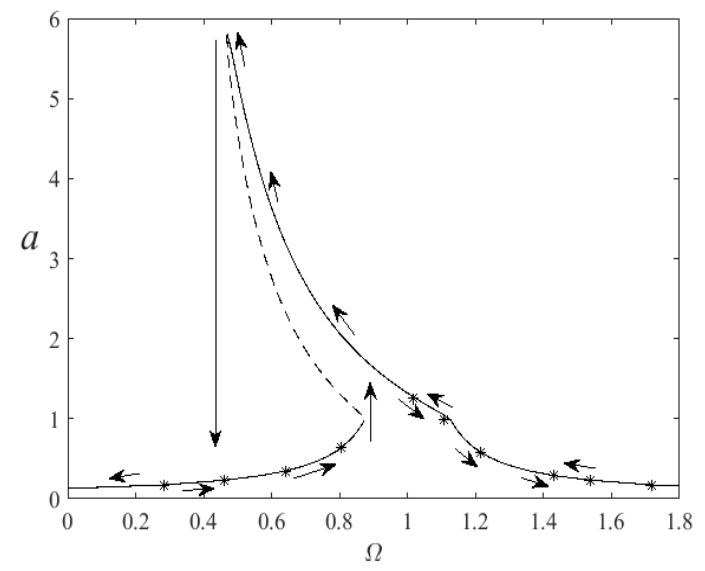

Fig.3 Frequency-amplitude response curve for $E=0.25, \beta=0.5, \alpha=0.05, X_{y}=1.0$ 


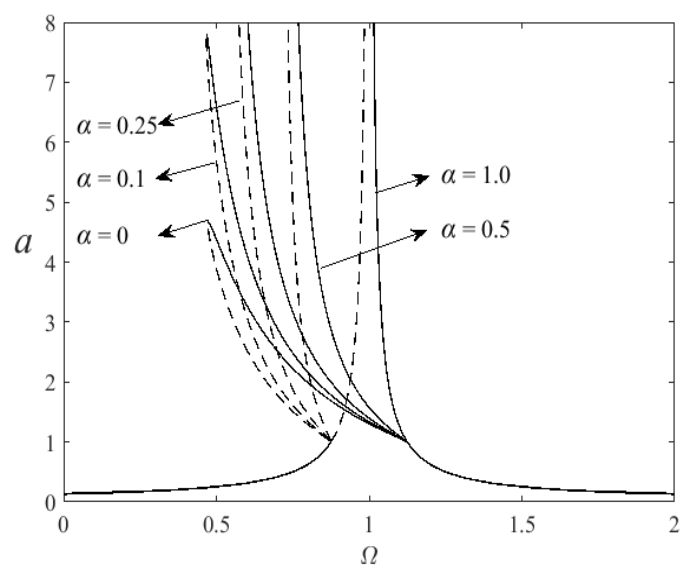

Fig.4 The effect of post-yielding stiffness coefficient $\alpha$ on frequency-amplitude response curve under $E=0.25, \beta=0.5, X_{y}=1.0$

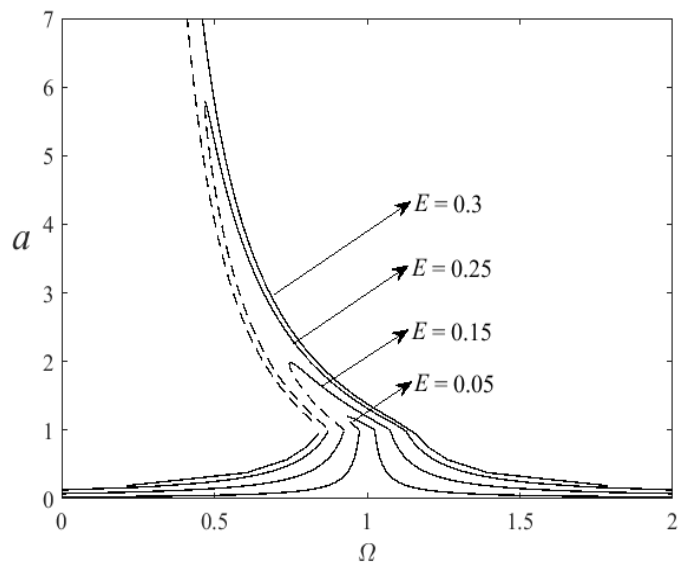

Fig.5 The effect of amplitude of harmonic excitation $E$ on frequency-amplitude response curve under $\beta=0.5, \alpha=0.05, X_{y}=1.0$

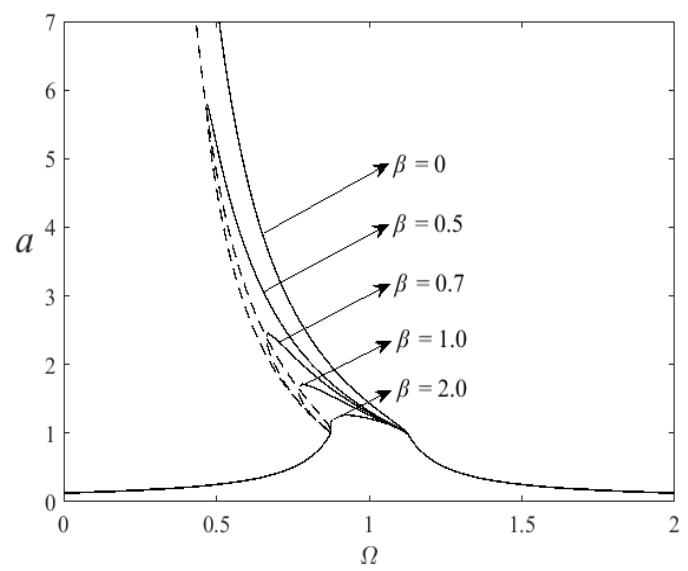

Fig.6 The effect of energy dissipation coefficient $\beta$ on frequency-amplitude response curve under $E=0.25, \alpha=0.05, X_{y}=1.0$

In Fig.4, the effect of post-yielding stiffness coefficient $\alpha$ is examined. The amplitude response is suppressed and the "soft" system characteristic becomes evident with the decrease of $\alpha$. At the same time, the deterministic jump phenomenon occurs in all cases except for $\alpha=1.0$. Similarly, Figs.5-6 illustrate the effect of amplitude of harmonic excitation $E$ and energy dissipation coefficient $\beta$, respectively. It can be perceived that the increase of $\beta$ or decrease of $E$ can contribute to smaller 
value amplitude response, but narrower the range of triple-valued region at which the corresponding deterministic jump takes place. In addition, such phenomenon is more obvious when $\beta$ is less than 0.7 and $E$ is more than 0.15 .

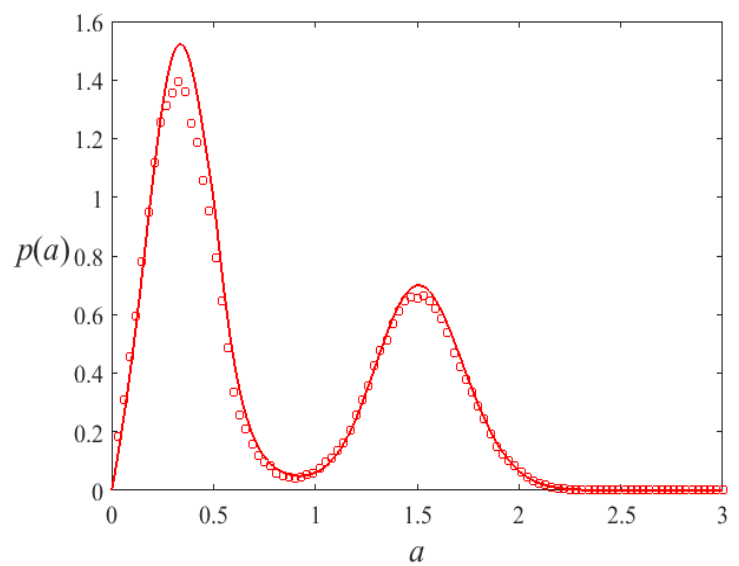

Fig.7 The stationary probability density $p(a)$ of the flag-shaped hysteresis system excited by combined harmonic and Gaussian white noise when $E=0.21, c=0.1, \alpha=0.05, \beta=0.5, X_{y}=0.5, \Omega=0.65, D_{1}=0.003$. Solid line denotes the analytical result; symbol denotes the result from Monte Carlo simulation.

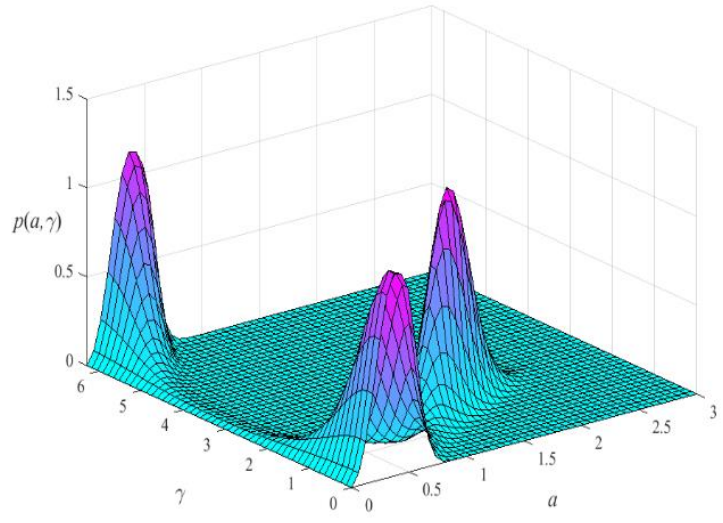

Fig.8 The surface plot of stationary joint probability density $p(a, \gamma)$ of the flag-shaped hysteresis system excited by combined harmonic and Gaussian white noise when $E=0.21, c=0.1, \alpha=0.05, \beta=0.5, X_{y}=0.5$, $\Omega=0.65, D_{1}=0.003$.

Next, the stochastic analysis of flag-shaped hysteretic system exposed to harmonic excitation and Gaussian white noise is studied. A classical response exhibiting bimodal shape is shown in Fig.7 and Fig. 8. It means that there are two stationary solutions and the stochastic jump may take place back and forth within the two peaks. It should be pointed out that there is noteworthy difference between stochastic jump and deterministic jump [32]. The latter takes place only on the left or right side of the three-valued region. 


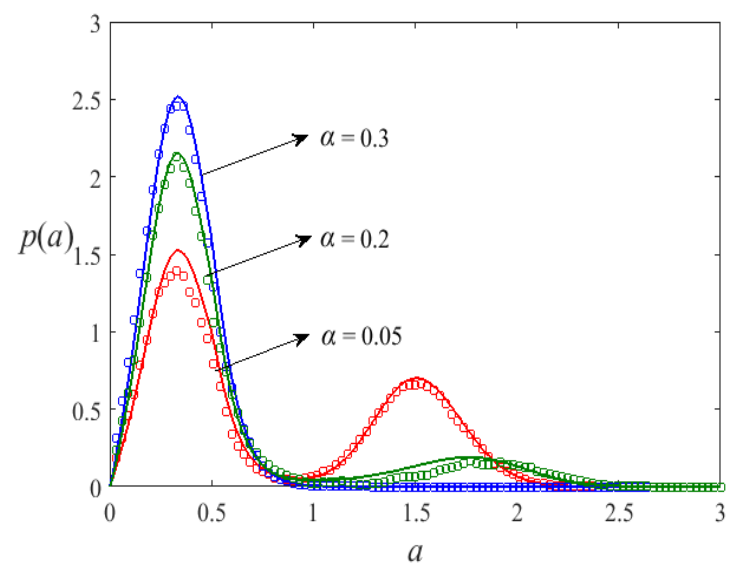

Fig.9 The stationary probability density $p(a)$ of the flag-shaped hysteresis system excited by combined harmonic and Gaussian white noise at different values of $\alpha$. The other parameters are set as $E=0.21$, $c=0.1, \beta=0.5, X_{y}=0.5, \Omega=0.65, D_{1}=0.003$. Solid line denotes the analytical result; symbol denotes the result from Monte Carlo simulation.

Fig.9 shows the stationary PDFs at different value of $\alpha$. It can be seen that the stochastic jump may occur again as $\alpha$ approaches to 0.05 . Besides, as $\alpha$ is increasing from 0.05 to 0.3 , the bimodal shape becomes unimodal and stochastic P-bifurcation takes place. Such similar phenomenon can be detected in Fig.10 as well. With the increase of $\beta$ from 0.5 to 1.0 , the stochastic P-bifurcation occurs and the stochastic jump takes place when $\beta=0.7$ and $\beta=0.5$. Moreover, the stochastic jump and P-bifurcation of system response both can be observed at different values for $X_{y}$ (see Fig.11), $E$ (see Fig.12) and $\Omega$ (see Fig.13), respectively.

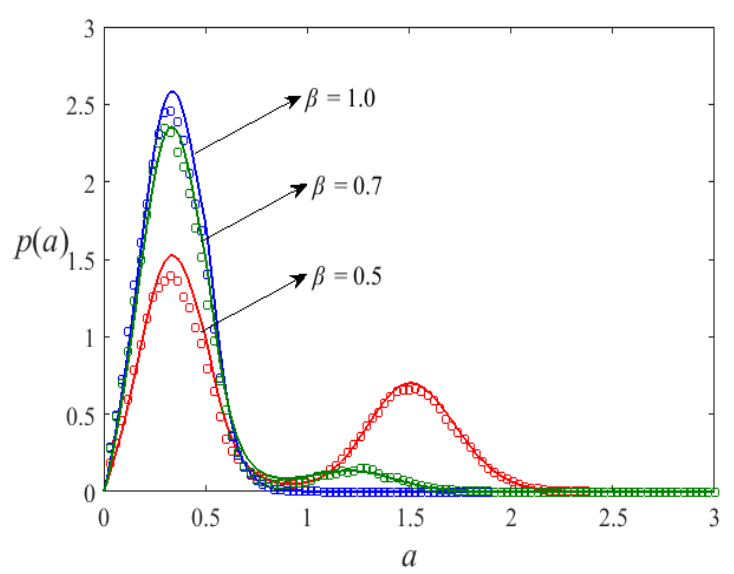

Fig.10 The stationary probability density $p(a)$ of the flag-shaped hysteresis system excited by combined harmonic and Gaussian white noise at different values of $\beta$. The other parameters are set as $E=0.21, c=0.1, \alpha=0.05, X_{y}=0.5, \Omega=0.65, D_{1}=0.003$. Solid line denotes the analytical result; symbol denotes the result from Monte Carlo simulation. 


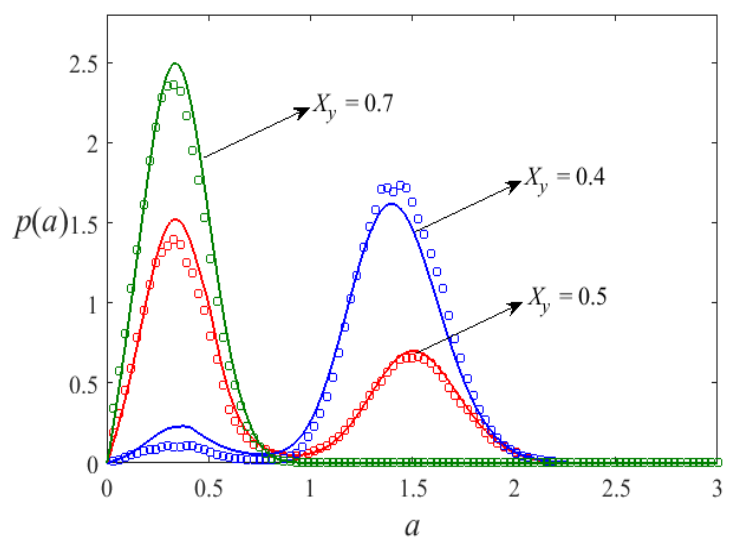

Fig.11 The stationary probability density $p(a)$ of the flag-shaped hysteresis system excited by combined harmonic and Gaussian white noise at different values of $X_{y}$. The other parameters are set as $E=0.21$, $c=0.1, \alpha=0.05, \beta=0.5, \Omega=0.65, D_{1}=0.003$. Solid line denotes the analytical result; symbol denotes the result from Monte Carlo simulation.

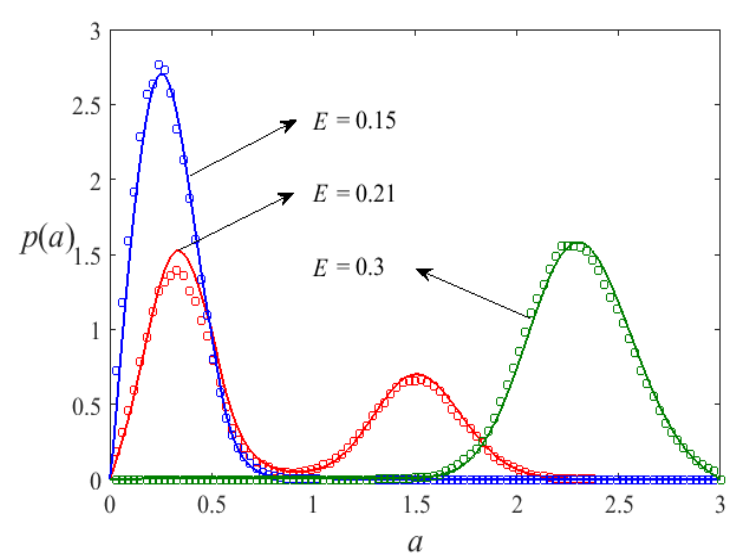

Fig.12 The stationary probability density $p(a)$ of the flag-shaped hysteresis system excited by combined harmonic and Gaussian white noise at different values of $E$. The other parameters are set as $c=0.1, \alpha=0.05, \beta=0.5, X_{y}=0.5, \Omega=0.65, D 1=0.003$. Solid line denotes the analytical result; symbol denotes the result from Monte Carlo simulation.

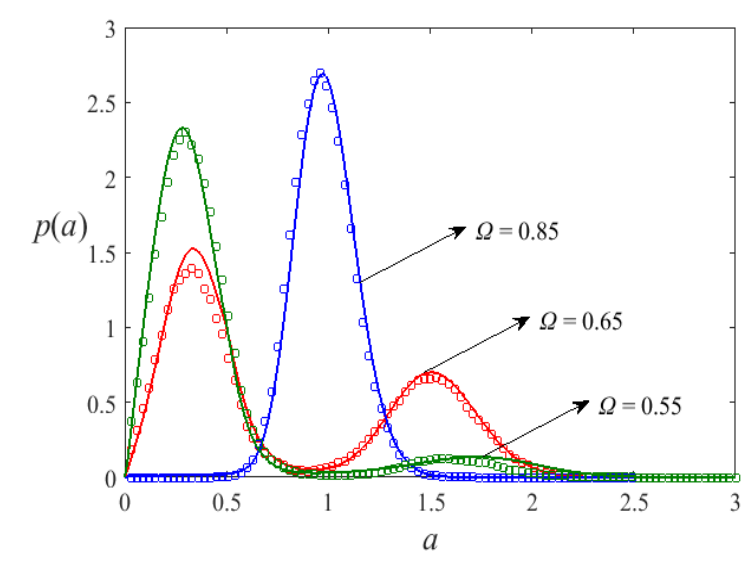

Fig.13 The stationary probability density $p(a)$ of the flag-shaped hysteresis system excited by combined harmonic and Gaussian white noise at different values of $\Omega$. The other parameters are set as $E=0.21, c=0.1, \alpha=0.05, \beta=0.5, X_{y}=0.5, D_{1}=0.003$. Solid line denotes the analytical result; symbol denotes the result from Monte Carlo simulation. 
For comparison, the non-resonance response of the same system is also given below (see Figs. 14-15). Fig.14 present the comparison of resonance and non-resonance responses at difference value of $E$. Similarly, the comparison of the two responses at difference value of $\Omega$ are shown in Fig. 15. All results show that the non-resonance response is evidently smaller than that of the resonance response under any parameters.

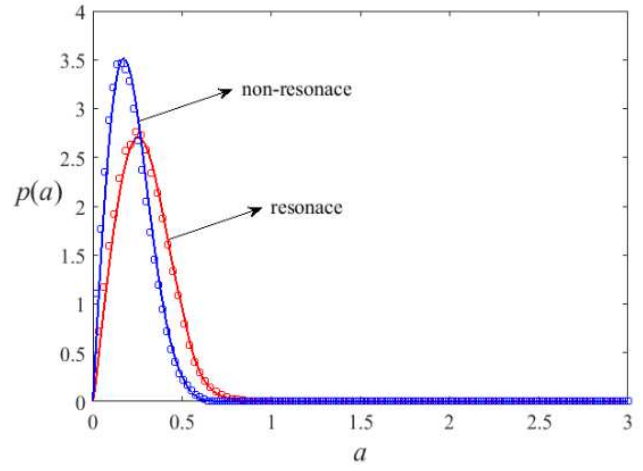

Fig.14a. $E=0.15$

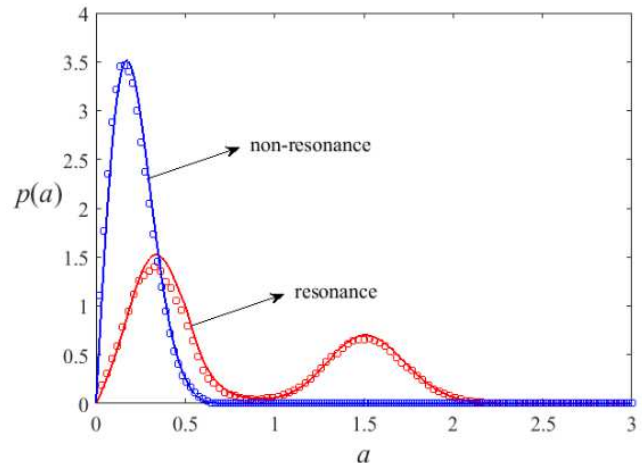

Fig.14b. $E=0.21$

Fig.14 Comparison between resonance and non-resonance case at different values of $E$. The other parameters are $c=0.1, \alpha=0.05, D_{1}=0.003, \beta=0.5, X_{y}=0.5, \Omega=0.65$. Solid line denotes the analytical result; symbol denotes the result from Monte Carlo simulation.

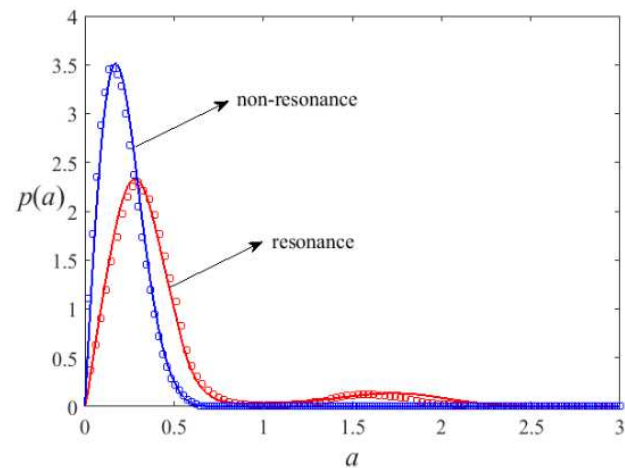

Fig.15a $\Omega=0.55$

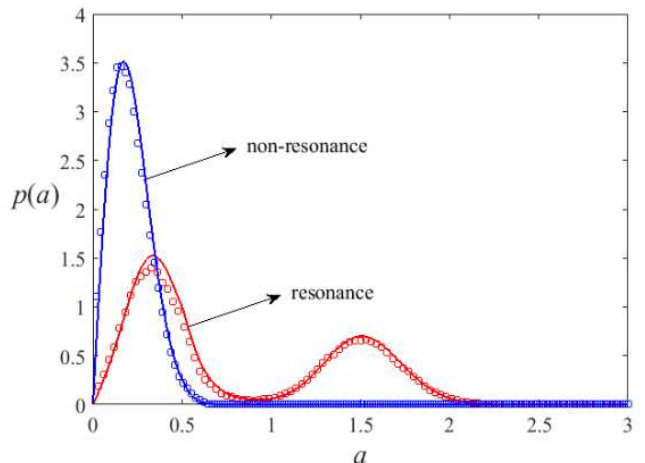

Fig.15b $\Omega=0.65$

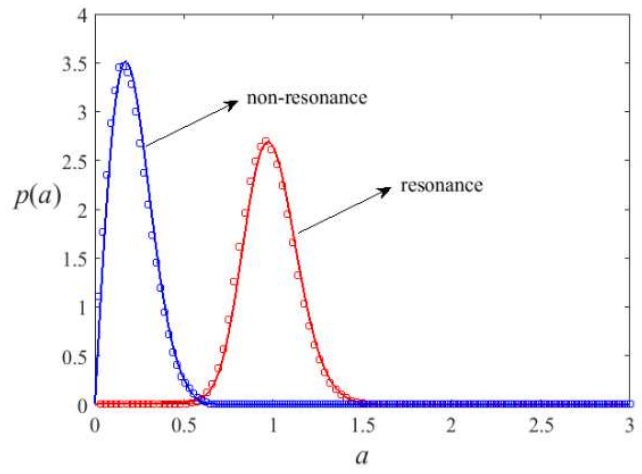

Fig.15c $\Omega=0.85$

Fig.15 Comparison between resonance and non-resonance case at different values of $\Omega$. The other parameters are set as $E=0.21, c=0.1, \alpha=0.05, D_{1}=0.003, \beta=0.5, X_{y}=0.5$. Solid line denotes the analytical result; symbol denotes the result from Monte Carlo simulation. 


\section{Conclusion}

In this paper, the stationary response of both harmonic and Gaussian white noise excited flag-shaped hysteretic system has been investigated. Considering of the resonance case, the effect of pure harmonic excitation has been examined firstly. The typical "soft" system behavior and deterministic jump phenomenon of flag-shaped hysteresis system have been observed. When the Gaussian white noise is added, the stationary PDF displays as apparent bimodal shape in a certain combination of the parameters and the typical stochastic jump may take place. The significant P-bifurcation phenomena have been examined with the change of system significant parameters, including the frequency and amplitude of harmonic excitation, post-yielding stiffness coefficient energy dissipation coefficient, yield displacement. Besides, the non-resonance case has been also investigated for comparison. Numerical results show that the non-resonance response is always smaller than that of resonance response. Given that the practical engineering often encounters with both two kinds of excitations, there is a great need to avoid the stochastic jump and P-bifurcation phenomenon, which will cause harm to the system and further destruct the structure.

\section{Acknowledgment}

This work is supported by the National Natural Science Foundation of China (No. 12072118), the Natural Science Foundation of Fujian Province of China (No.2019J01049), the Project for Youth Innovation Fund of Xiamen (No. 3502Z20206005), and the Project for Postgraduates' Innovative Fund in Scientific Research of Huaqiao University.

\section{Compliance with ethical standards}

\section{Conflict of interest}

The authors declare that they have no conflict of interest

\section{References}

[1] Cai, G. Q., Lin, Y. K.: On randomly excited hysteretic structures. J. Appl. Mech. 57, 442-448 (1990)

[2] Roohi, M., Erazo, K., Rosowsky, D., Hernandez, E. M.: An extended model-based observer for state estimation in nonlinear hysteretic structural systems. Mech. Syst. Signal Pr. 146, $107015(2020)$

[3] Ozbulut, O. E., Hurlebaus, S.: Re-centering variable friction device for vibration control of structures subjected to near-field earthquakes. Mech. Syst. Signal Pr. 25, 2849-2862 (2011)

[4] Grigorian, M., Moghadam, A. S., Mohammadi, H., kamizi, M.: Methodology for developing 
earthquake resilient structures. Struct. Design Tall Spec. Build. 28, 1-20 (2019)

[5] Christopoulos, C.: Frequency response of flag-shaped single-degree-of-freedom hysteretic systems. J. Eng. Mech. 130, 894-903 (2004)

[6] Gur, S., Mishra, S. K., Roy, K.: Stochastic seismic response of building with super-elastic damper. Mech. Syst. Signal Pr. 72-73, 642-659 (2016)

[7] Dobson, S., Noori, M., Hou, Z., Dimentberg, M., Baber, T.: Modeling and random vibration analysis of SDOF systems with asymmetric hysteresis. Int. J. Non-Lin. Mech. 32(4), 669-680 (1997)

[8] Kashan, H.: Analytical parametric study of bi-linear hysteretic model of dry friction under harmonic, impulse and random excitations. Nonlinear Dynam. 89(1), 267-279 (2017)

[9] Quaranta, G., Mollaioli, F.: On the use of the equivalent linearization for bilinear oscillators under pulse-like ground motion. Eng. Struct. 160, 395-407 (2018)

[10] Spanos, P. D., Matteo, A. D., Pirrotta, A.: Steady-state dynamic response of various hysteretic systems endowed with fractional derivative elements. Nonlinear Dynam. 98, 3113-3124 (2019)

[11] Colangelo, F.: Interaction of axial force and bending moment by using Bouc-Wen hysteresis and stochastic linearization. Struct. Saf. 67, 39-53 (2017)

[12] Su, C., Xian, J., Huang, H.: An iterative equivalent linearization approach for stochastic sensitivity analysis of hysteretic systems under seismic excitations based on explicit time-domain method. Comput. Struct. 242, 106396 (2020)

[13] Xu, J., Wang, J., Wang, D.: Evaluation of the probability distribution of the extreme value of the response of nonlinear structures subjected to fully non-stationary stochastic seismic excitations. J. Eng. Mech. 146(2) (2019)

[14] Chen, J., Yuan, S.: Pdem-based dimension-reduction of fpk equation for additively excited hysteretic nonlinear systems. Probabilistic Eng. Mech. 38, 111-118 (2014)

[15] Waubke, H., Kasess, C. H.: Gaussian closure technique applied to the hysteretic Bouc model with non-zero mean white noise excitation. J. Sound Vib. 382, 258-273 (2016)

[16] Guo, S. S.: Nonstationary solutions of nonlinear dynamical systems excited by Gaussian white noise. Nonlinear Dynam. 92, 613-626 (2018)

[17] Guo, S. S., Shi, Q. X., Xu, Z. D.: Nonstationary seismic responses of nonlinear structural systems to modulated earthquake excitations. J. Eng. Mech. 145(12) (2019)

[18] Yazdani, A., Salimi, M. R.: Earthquake response spectra estimation of bilinear hysteretic systems using random-vibration theory method. Earthq. Struct. 8(5), 1055-1067 (2015)

[19] Spanos, P. D., Cacciola, P., Muscolino, G.: Stochastic Averaging of Preisach Hysteretic Systems. J. Eng. Mech. 130(11), 1257-1267 (2004)

[20] Jin, X. L., Wang, Y., Huang, Z. L.: Analysis and control for transient responses of seismic-excited hysteretic structures. Soil Dyn. Earthq. Eng. 73, 58-65 (2015)

[21] Di Matteo, A., Spanos, P. D., Pirrotta, A.: Approximate survival probability determination of 
hysteretic systems with fractional derivative elements. Probabilistic Eng. Mech. 54, 138-146 (2018)

[22] Zeng, Y., Li, G.: Stationary response of bilinear hysteretic system driven by Poisson white noise. Probabilistic Eng. Mech. 33(jul.), 135-143 (2013)

[23] Ying, Z. G., Zhu, W. Q., Ni, Y. Q., Ko, J. M.: Stochastic averaging of duhem hysteretic systems. J. Sound Vib. 254(1), 91-104 (2002)

[24] Ni, Y. Q., Ying, Z. G., Ko, J. M., Zhu, W. Q.: Random response of integrable Duhem hysteretic systems under non-white excitation. Int. J. Non-Lin. Mech. 37(8), 1407-1419 (2002)

[25] Wang, Y., Ying, Z. G., Zhu, W. Q.: Stochastic averaging of energy envelope of preisach hysteretic systems. J. Sound Vib. 321(3-5), 976-993 (2009)

[26] Spanos, P. D., Kontsos, A., Cacciola, P.: Steady-state dynamic response of preisach hysteretic systems. J. Vib. Acoust. 128(2), 1457-1466 (2006)

[27] Kim, T., Kwon, O. S., Song, J.: Response prediction of nonlinear hysteretic systems by deep neural networks. Neural Networks. 111, 1-10 (2018)

[28] Pradlwarter, H. J., Schueller, G. I., Dorka, U.: Reliability of mdof-systems with hysteretic devices. Eng. Struct. 20(8), 685-691 (1998)

[29] Zhu, W. Q.: Stochastic averaging methods in random vibration. Appl. Mech. Rev. 41, 189-199 (1988)

[30] Hu, X. B., Jiang, W. B.: A random seismic response analysis of self-centering single degree of freedom system. J. Sound Vib. 35, 152-157 (2016) (In Chinese)

[31] Zhang, H. M., Quan, L. M., Lu, X. L., Xu, J. Q.: Modified flag-shaped model for self-centering system and its equivalent linearization and structural optimization for stochastic excitation. Eng. Struct. 215, 110420 (2020)

[32] Zhu, W. Q., Lu, M. Q., Wu, Q. T.: Stochastic jump and bifurcation of a duffing oscillator under narrow-band excitation. J. Sound Vib. 165, 285-304 (1993) 
Figures

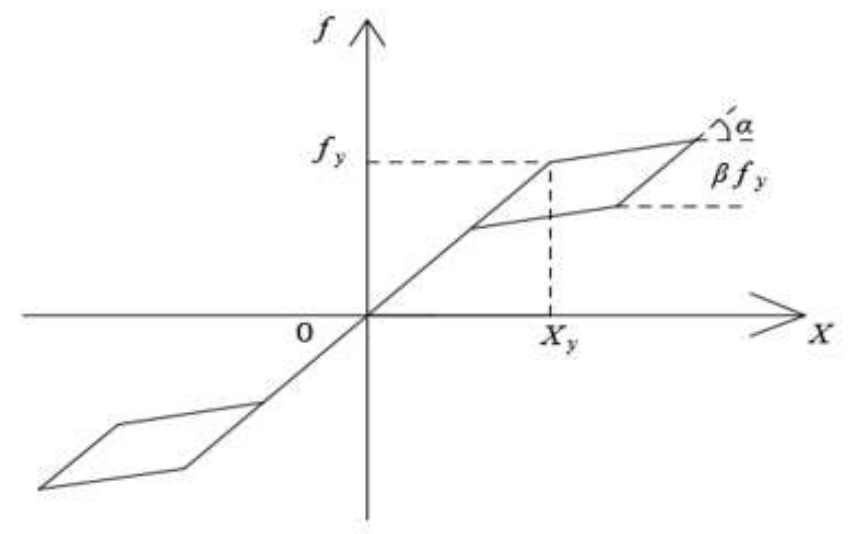

(a) The flag-shaped restoring force

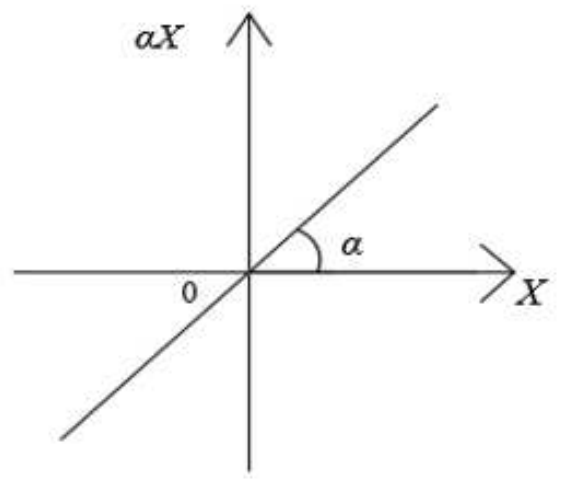

(b) The elastic force

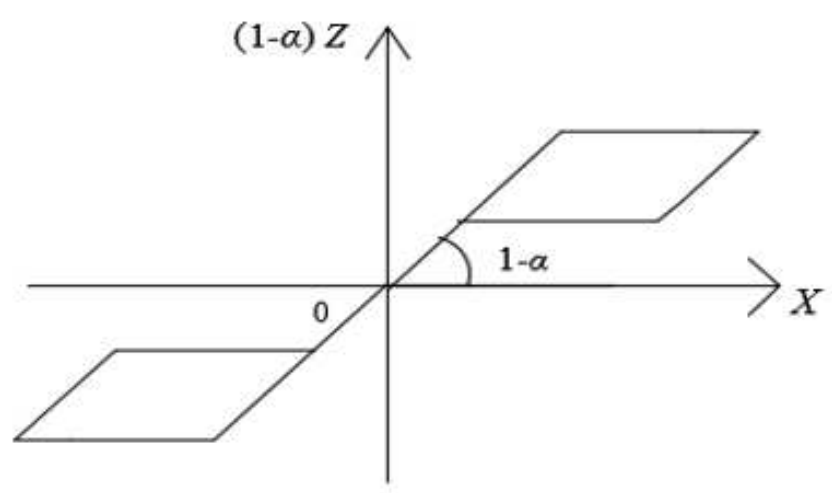

(c) The hysteretic force

Figure 1

The restoring force of the flag-shaped hysteretic system 


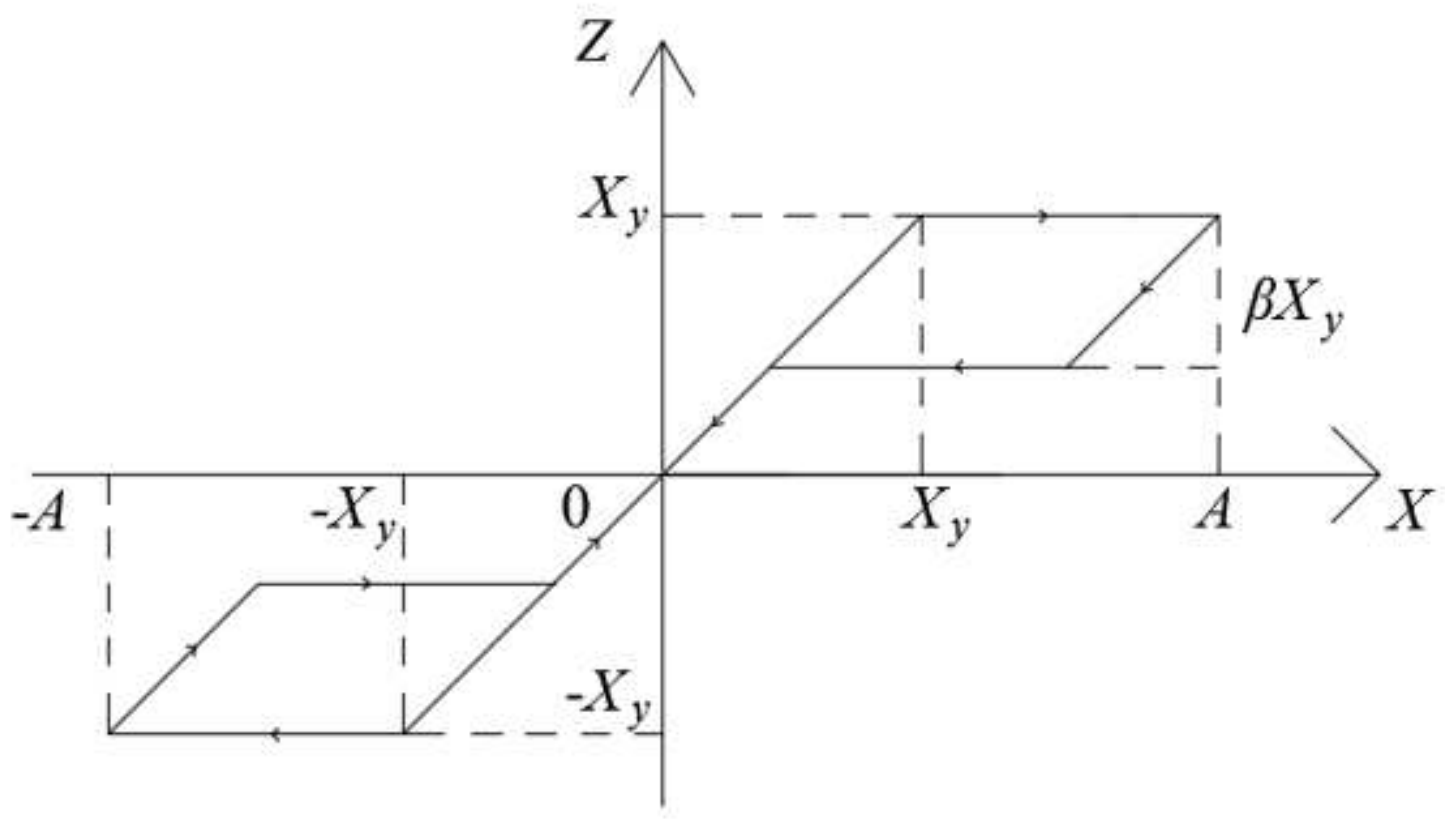

Figure 2

The hysteretic displacement of the flag-shaped hysteretic system 


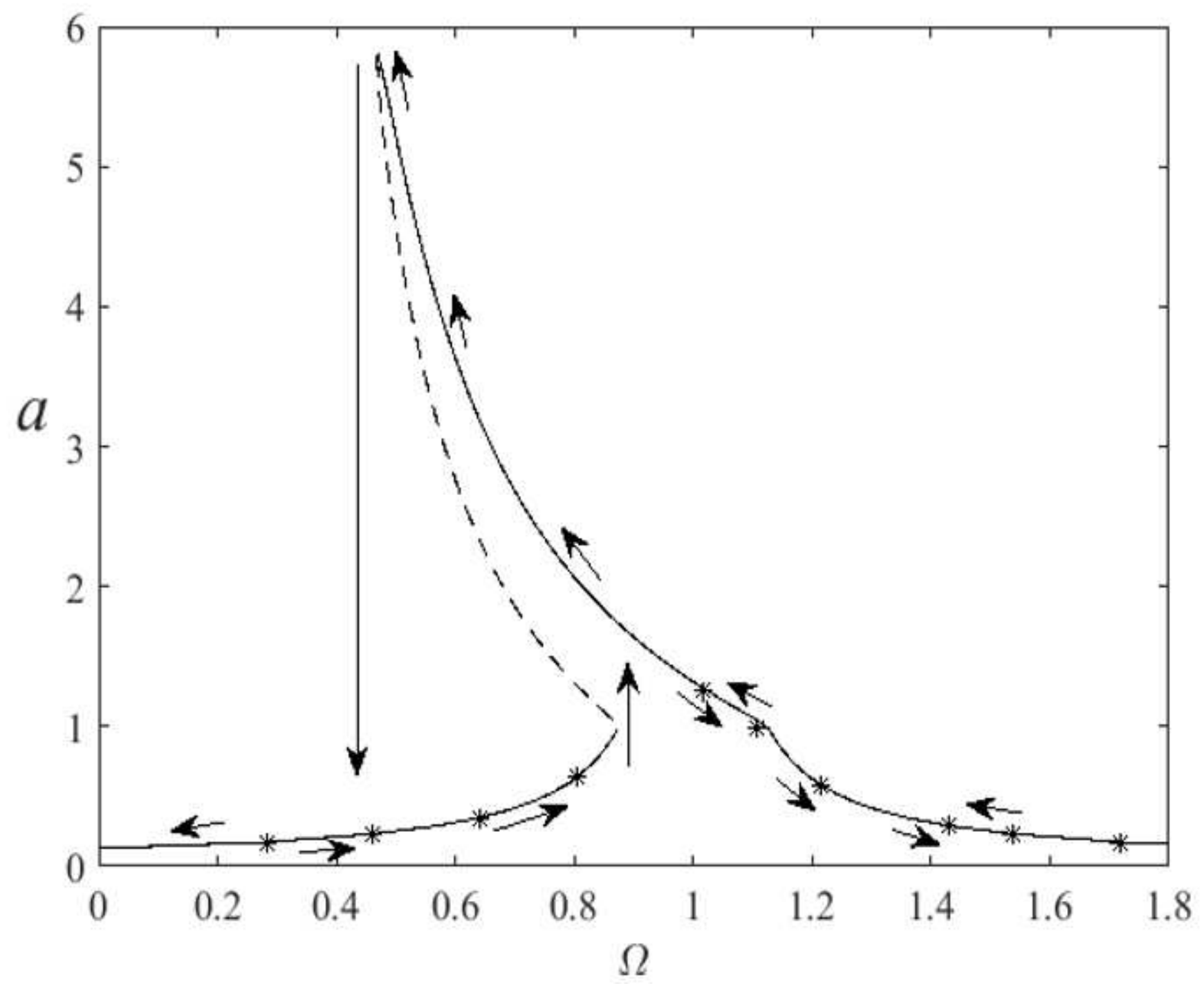

Figure 3

Frequency-amplitude response curve for $E=0.25, \beta=0.5, \alpha=0.05, X y=1.0$ 


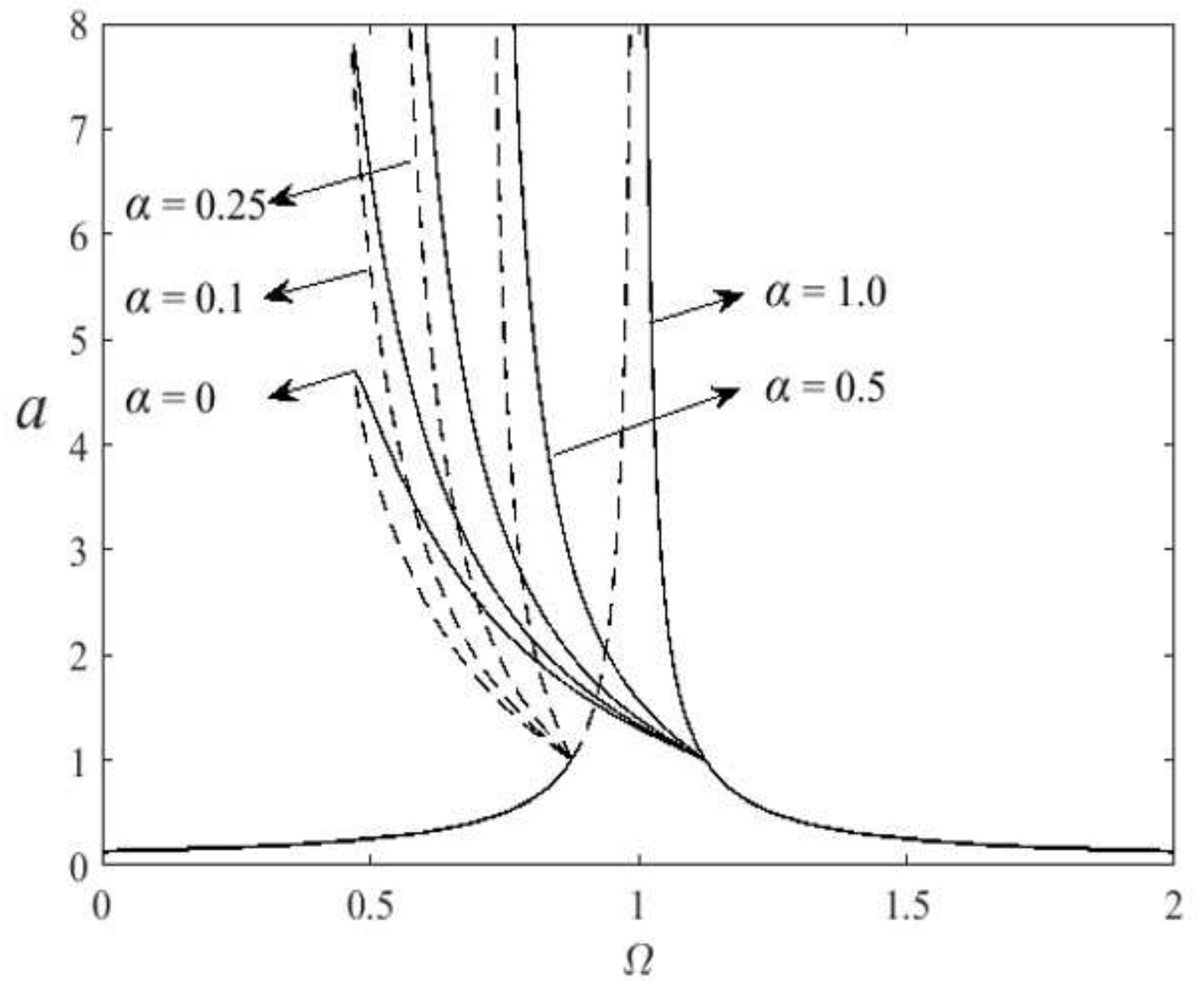

Figure 4

The effect of post-yielding stiffness coefficient $a$ on frequency-amplitude response curve under $E=0.25$, $\beta=0.5, X y=1.0$ 


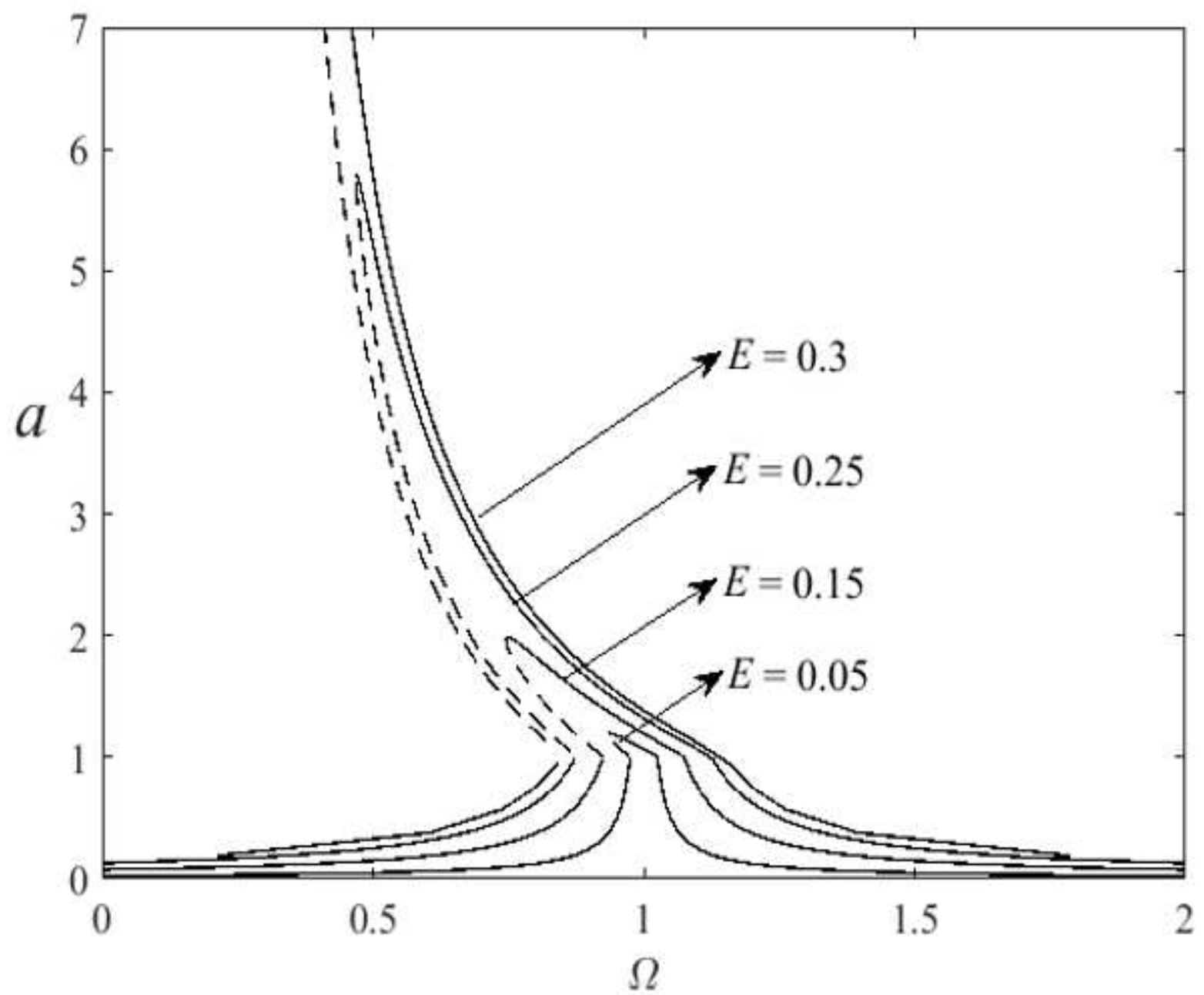

Figure 5

The effect of amplitude of harmonic excitation $E$ on frequency-amplitude response curve under $\beta=0.5$, $a=0.05, X y=1.0$ 


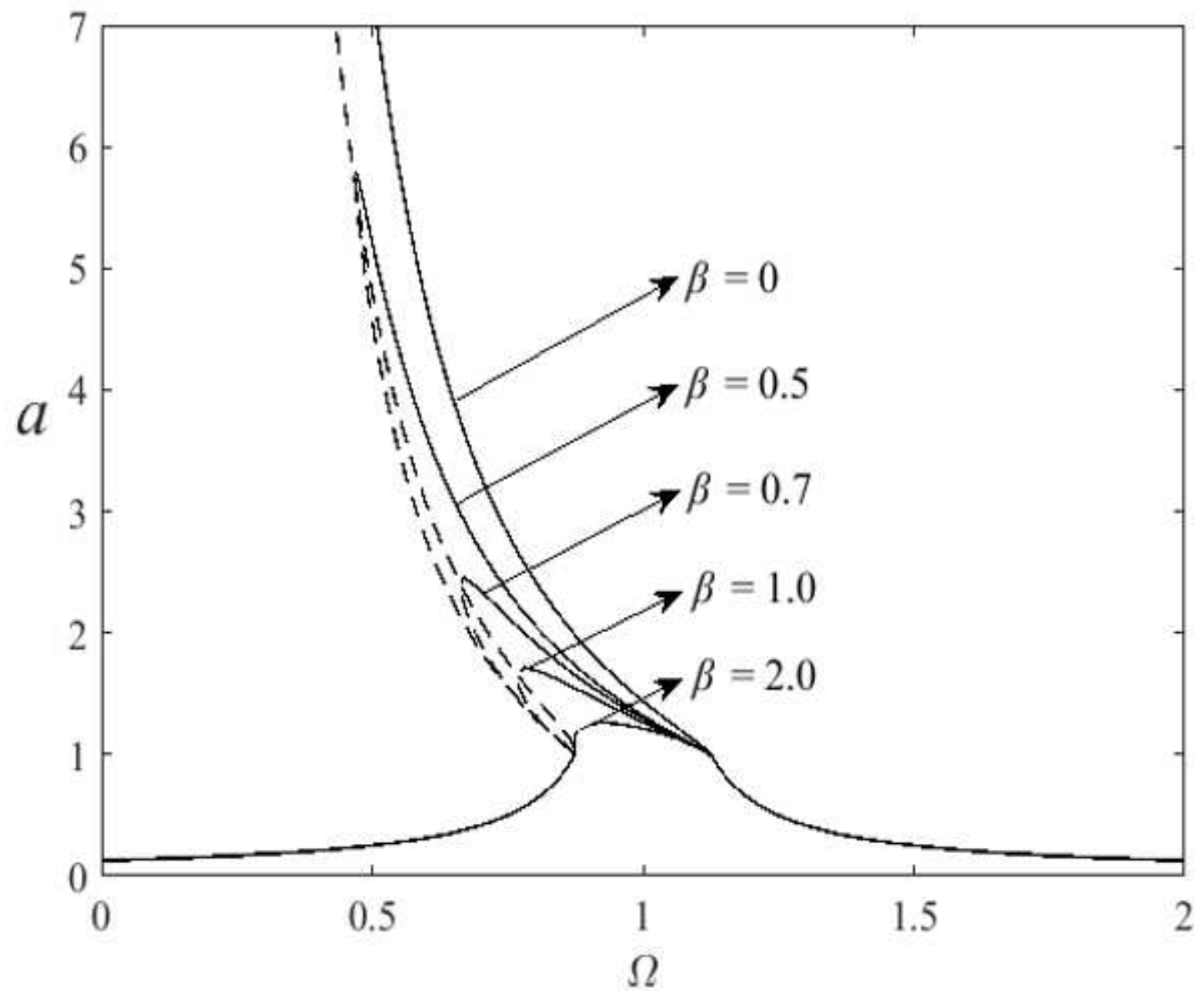

Figure 6

The effect of energy dissipation coefficient $\beta$ on frequency-amplitude response curve under $E=0.25$, $a=0.05, X y=1.0$ 


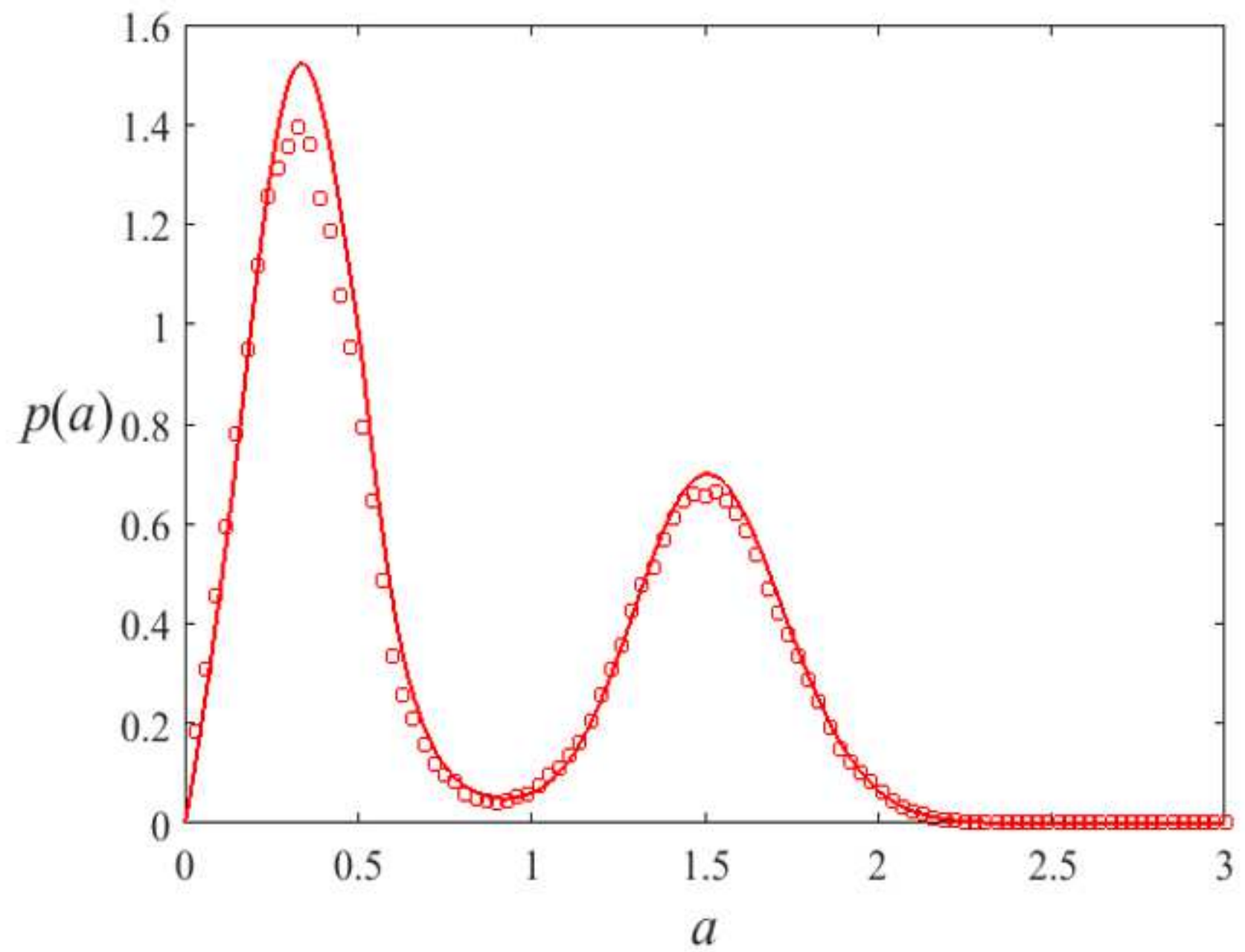

Figure 7

The stationary probability density $\mathrm{p}(\mathrm{a})$ of the flag-shaped hysteresis system excited by combined harmonic and Gaussian white noise when $E=0.21, c=0.1, a=0.05, \beta=0.5, X y=0.5, \Omega=0.65, D 1=0.003$. Solid line denotes the analytical result; symbol denotes the result from Monte Carlo simulation. 


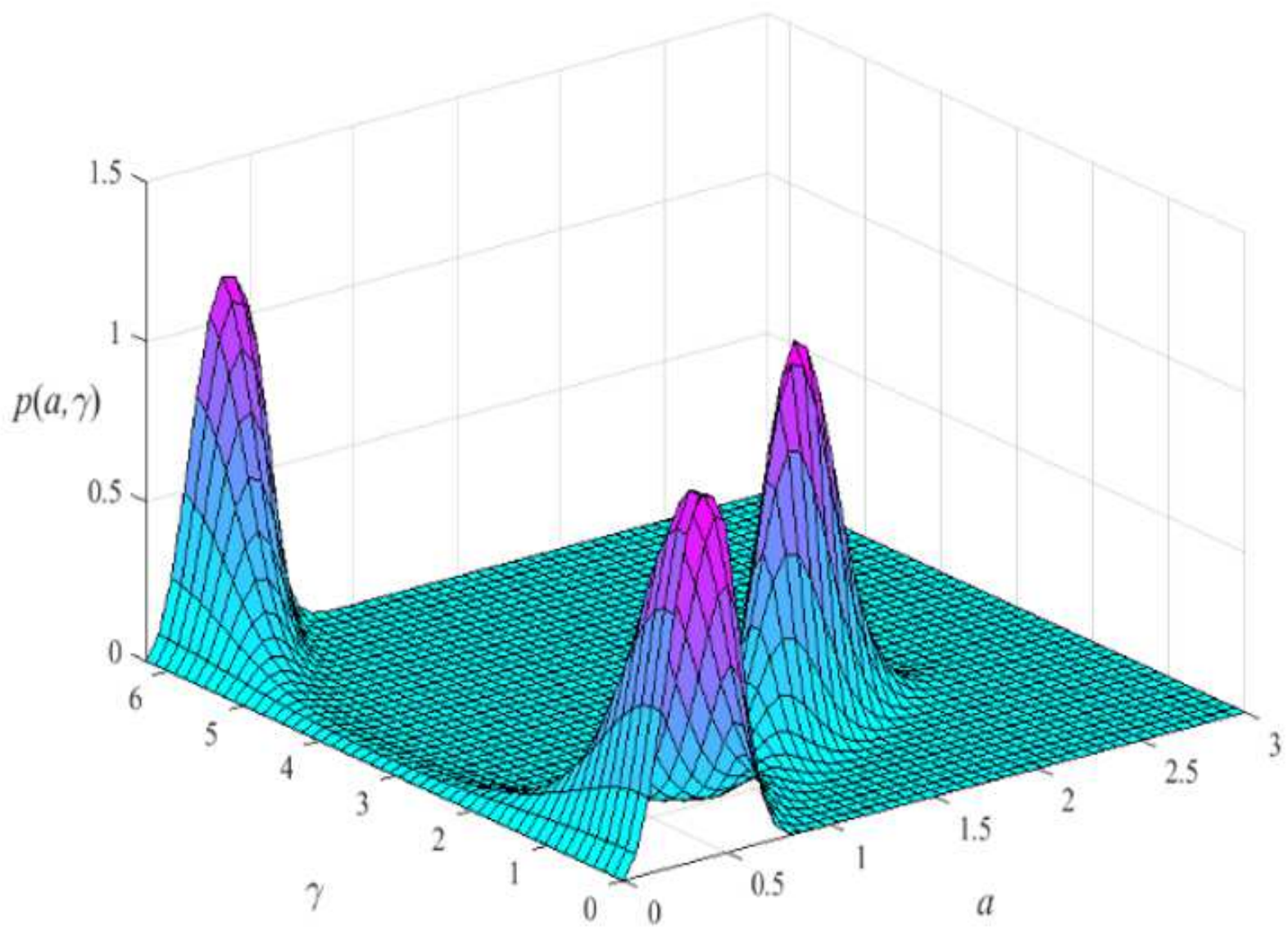

Figure 8

The surface plot of stationary joint probability density $p(a, y)$ of the flag-shaped hysteresis system excited by combined harmonic and Gaussian white noise when $E=0.21, c=0.1, a=0.05, \beta=0.5, X y=0.5, \Omega=0.65$, D1 $=0.003$. 


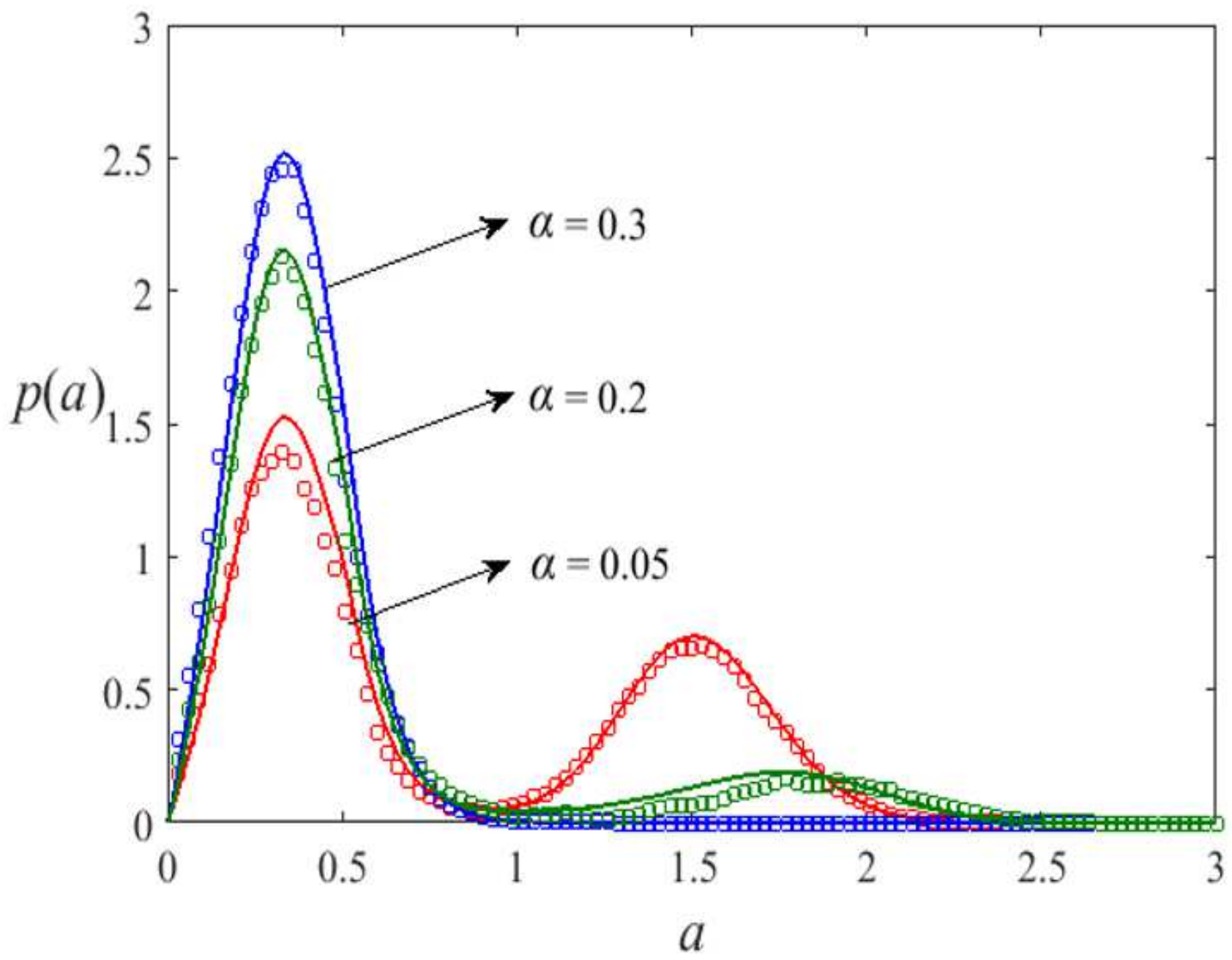

Figure 9

The stationary probability density $\mathrm{p}(\mathrm{a})$ of the flag-shaped hysteresis system excited by combined harmonic and Gaussian white noise at different values of $a$. The other parameters are set as $E=0.21$, $C=0.1, \beta=0.5, X y=0.5, \Omega=0.65, D 1=0.003$. Solid line denotes the analytical result; symbol denotes the result from Monte Carlo simulation. 


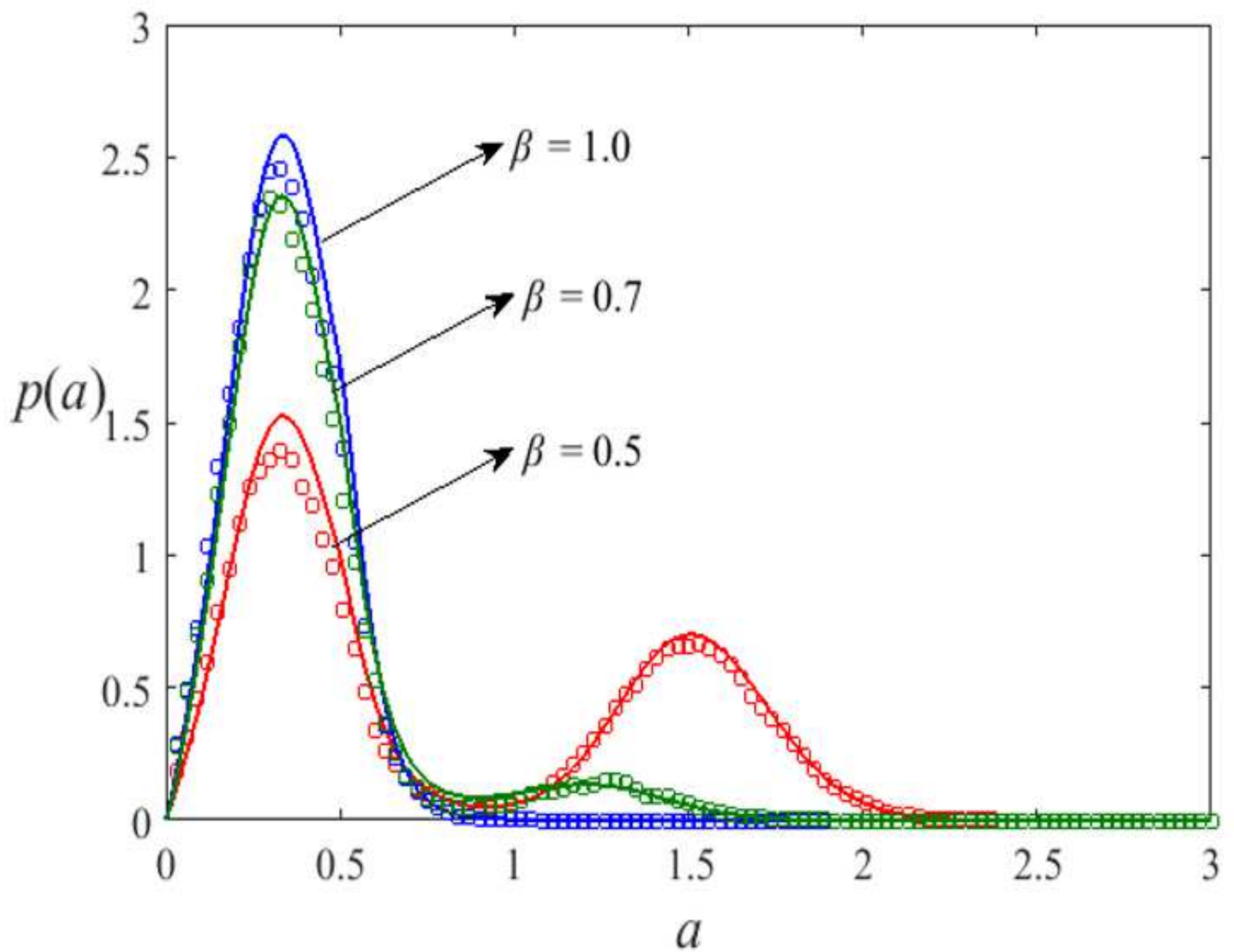

Figure 10

The stationary probability density $\mathrm{p}(\mathrm{a})$ of the flag-shaped hysteresis system excited by combined harmonic and Gaussian white noise at different values of $\beta$. The other parameters are set as $E=0.21$, $c=0.1, a=0.05, X y=0.5, \Omega=0.65, D 1=0.003$. Solid line denotes the analytical result; symbol denotes the result from Monte Carlo simulation. 


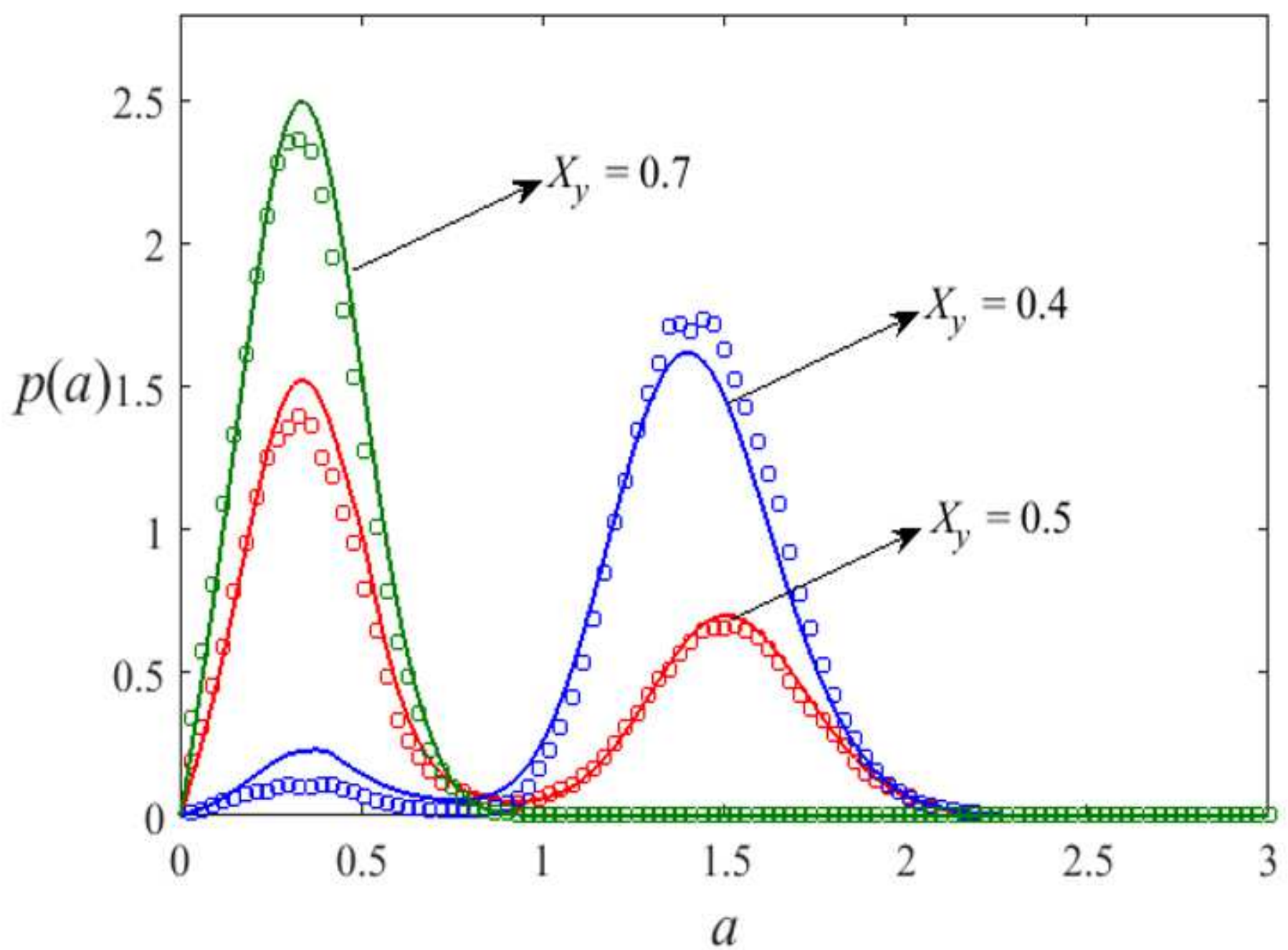

\section{Figure 11}

The stationary probability density $\mathrm{p}(\mathrm{a})$ of the flag-shaped hysteresis system excited by combined harmonic and Gaussian white noise at different values of $X y$. The other parameters are set as $E=0.21$, $c=0.1, a=0.05, \beta=0.5, \Omega=0.65, D 1=0.003$. Solid line denotes the analytical result; symbol denotes the result from Monte Carlo simulation. 


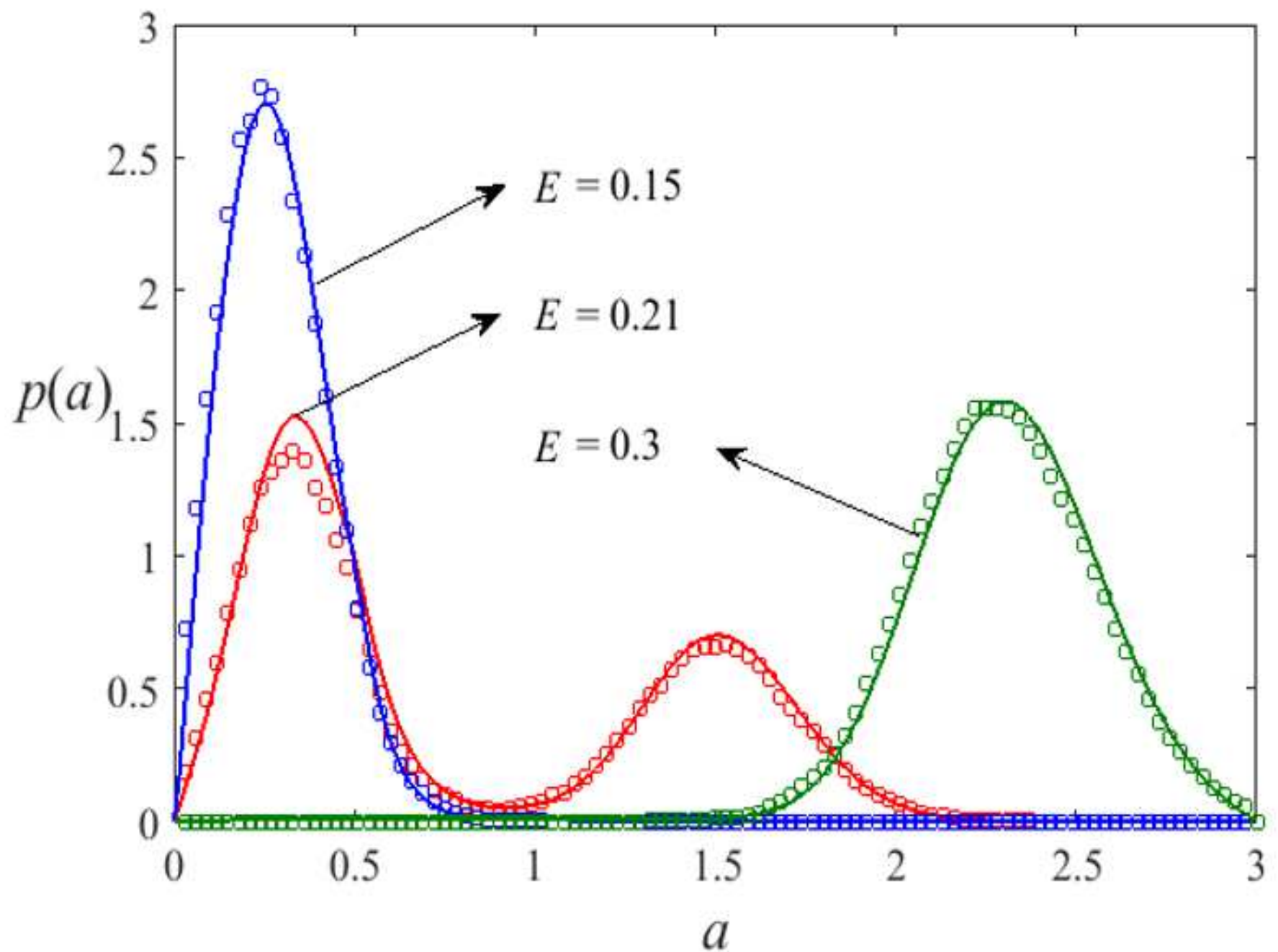

Figure 12

The stationary probability density $\mathrm{p}(\mathrm{a})$ of the flag-shaped hysteresis system excited by combined harmonic and Gaussian white noise at different values of $\mathrm{E}$. The other parameters are set as $\mathrm{c}=0.1$, $a=0.05, \beta=0.5, X y=0.5, \Omega=0.65, D 1=0.003$. Solid line denotes the analytical result; symbol denotes the result from Monte Carlo simulation. 


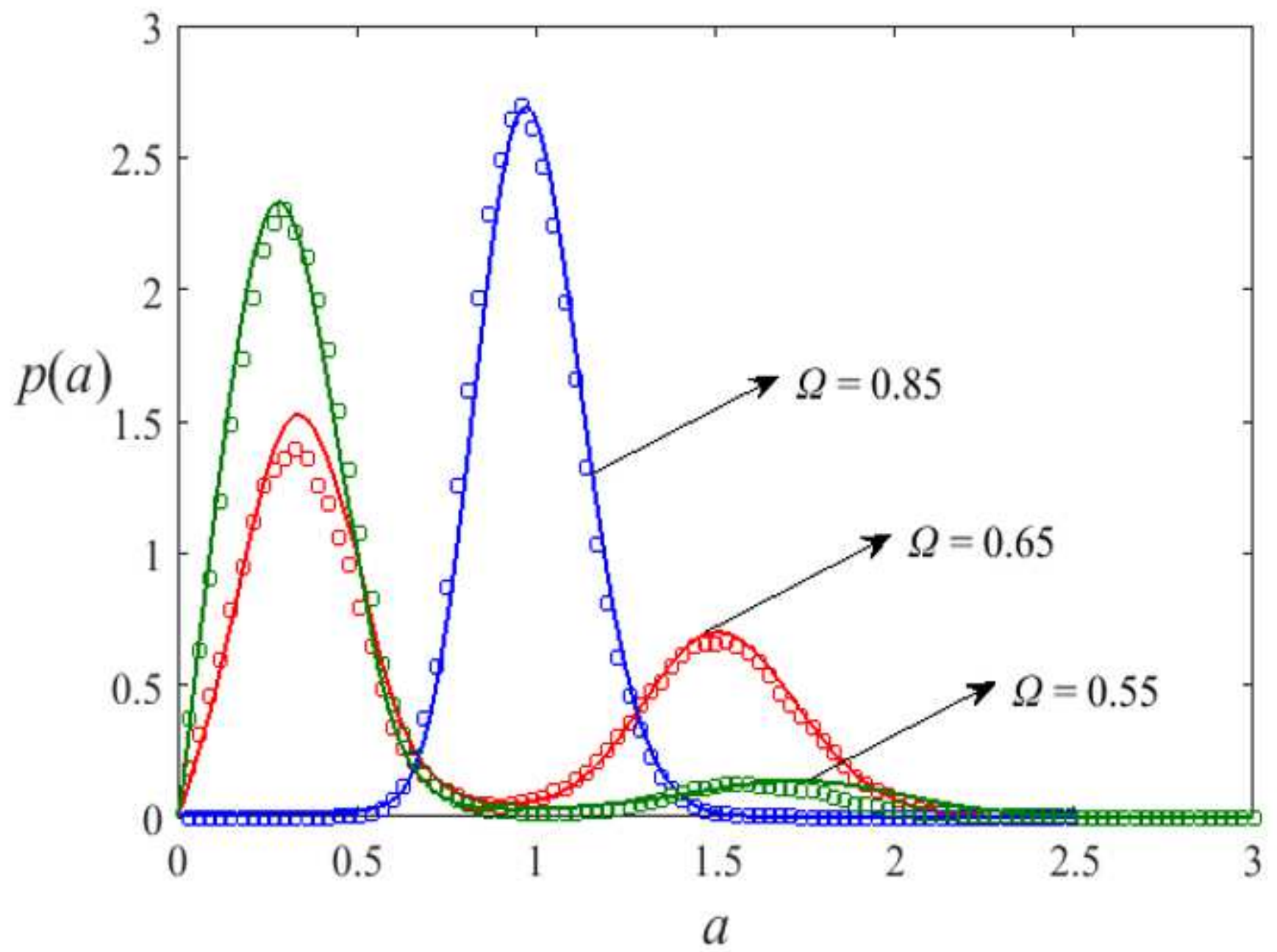

Figure 13

The stationary probability density $\mathrm{p}(\mathrm{a})$ of the flag-shaped hysteresis system excited by combined harmonic and Gaussian white noise at different values of $\Omega$. The other parameters are set as $E=0.21$, $c=0.1, a=0.05, \beta=0.5, X y=0.5, D 1=0.003$. Solid line denotes the analytical result; symbol denotes the result from Monte Carlo simulation.

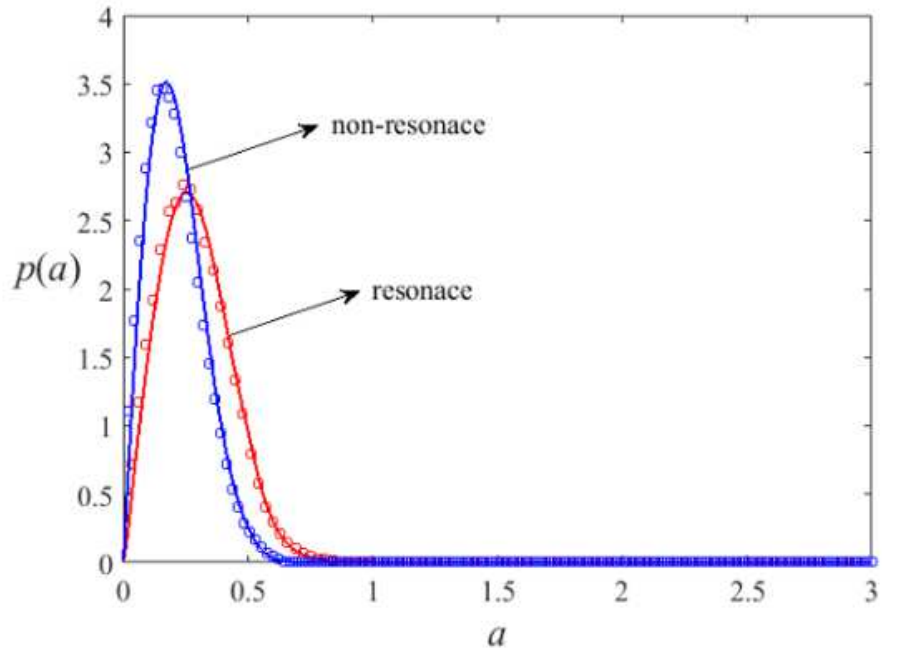

Fig.14a. $E=0.15$

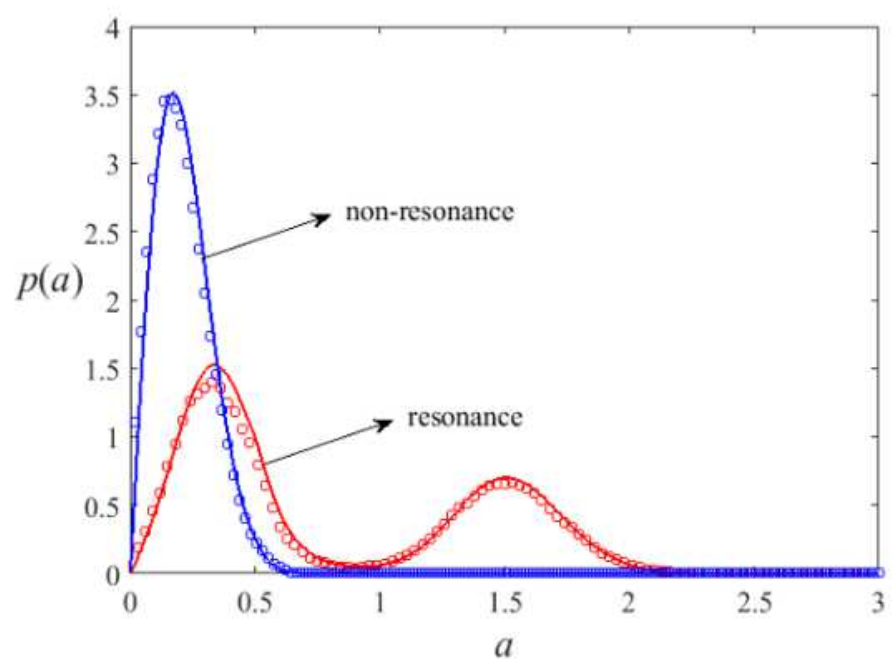

Fig.14b. $E=0.21$ 
Figure 14

Comparison between resonance and non-resonance case at different values of $E$. The other parameters are $c=0.1, a=0.05, D 1=0.003, \beta=0.5, X y=0.5, \Omega=0.65$. Solid line denotes the analytical result; symbol denotes the result from Monte Carlo simulation.

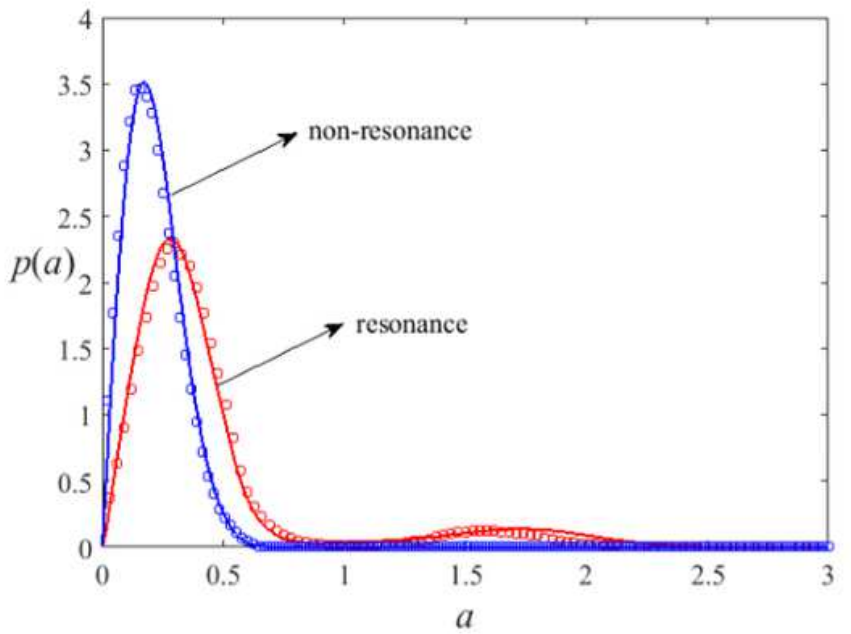

Fig.15a $\Omega=0.55$

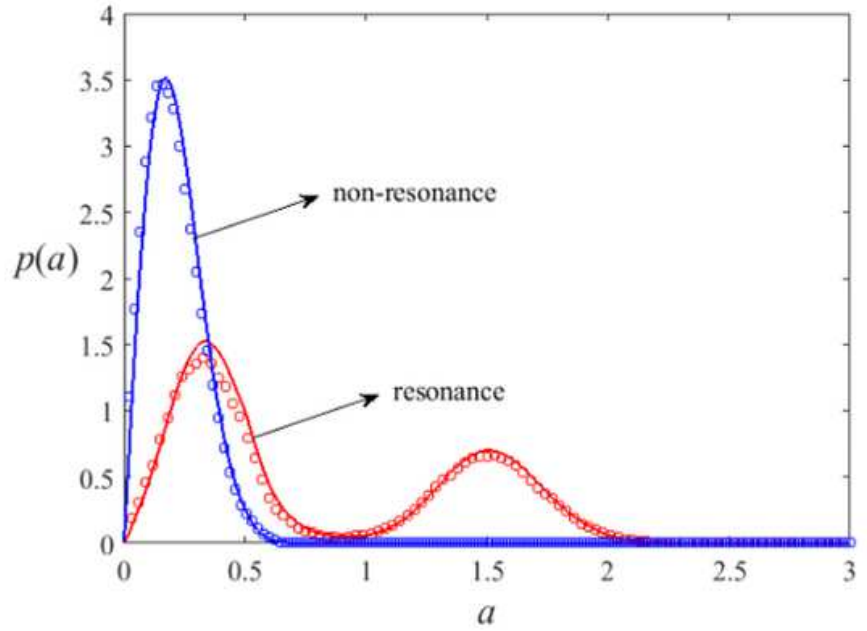

Fig.15b $\Omega=0.65$

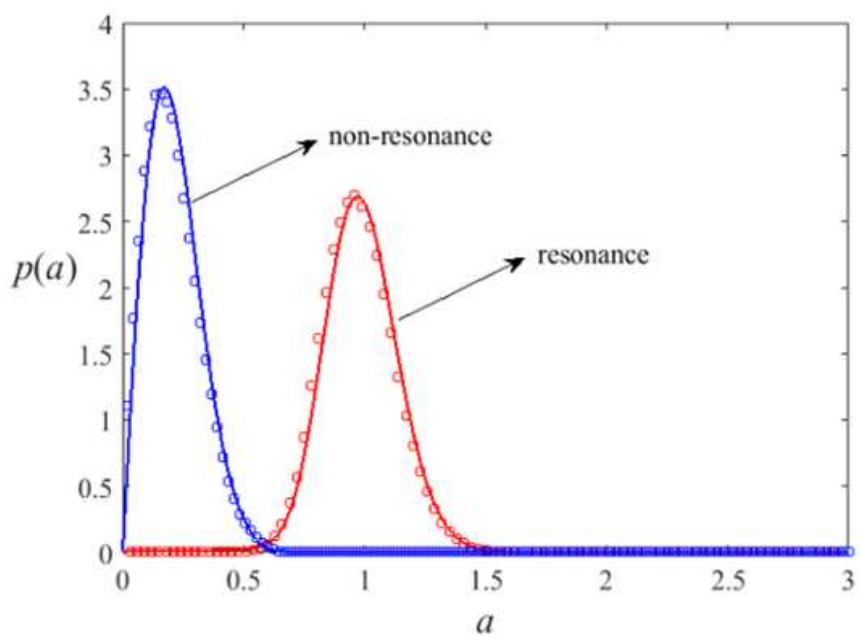

Fig.15c $\Omega=0.85$

\section{Figure 15}

Comparison between resonance and non-resonance case at different values of $\Omega$. The other parameters are set as $E=0.21, c=0.1, a=0.05, D 1=0.003, \beta=0.5, X y=0.5$. Solid line denotes the analytical result; symbol denotes the result from Monte Carlo simulation. 